\title{
The dimension-shift conjecture for one-loop amplitudes
}

\author{
Ruth Britto, ${ }^{a, b}$ Guy R. Jehu ${ }^{a}$ and Andrea Orta ${ }^{a}$ \\ ${ }^{a}$ School of Mathematics and Hamilton Mathematical Institute, Trinity College Dublin, \\ College Green, Dublin 2, Ireland \\ ${ }^{b}$ Institut de Physique Théorique, Université Paris Saclay, CEA, CNRS, \\ Gif-sur-Yvette cedex F-91191, France \\ E-mail: brittor@tcd.ie, jehu@maths.tcd.ie, andrea.orta.1988@gmail.com
}

Abstract: A conjecture made by Bern, Dixon, Dunbar, and Kosower asserts a simple dimension shifting relationship between the one-loop structure of $\mathcal{N}=4 \mathrm{MHV}$ amplitudes and all-plus helicity amplitudes in pure Yang-Mills theory. We prove this conjecture to all orders in dimensional regularisation using unitarity cuts, and evaluate the form of these simplest one-loop amplitudes using a generalised $D$-dimensional unitarity technique which captures the full amplitude to all multiplicities.

KEYwords: Scattering Amplitudes, Supersymmetric Gauge Theory, Gauge Symmetry

ArXIV EPRINT: 2011.13821 


\section{Contents}

1 Introduction $\quad 1$

1.1 Overview 3

2 Proof of the conjecture $\quad 7$

2.1 Unitarity cuts 8

2.2 Tree-level off-shell amplitudes $\quad 10$

$\begin{array}{ll}2.3 & \text { Proving the conjecture from the Coulomb branch } \\ \end{array}$

3 Generalised cuts and all-epsilon forms $\quad 17$

$\begin{array}{lll}3.1 & \text { Integral bases } & 17\end{array}$

3.2 The cut box 20

3.3 Pentagon coefficients 23

3.4 Six-point example 24

3.5 Reduction to finite limit 26

$\begin{array}{ll}3.6 & \text { Transcendental structure }\end{array}$

4 Conclusion and outlook $\quad 28$

\section{Introduction}

Planar $\mathcal{N}=4$ super Yang-Mills continues to prove itself as the simplest toy model to develop techniques for computing scattering amplitudes from their singular structure; recent work has pushed analytic ansätze for planar amplitudes up to seven points at four loops $[1,2]$. A remarkable feature which entails this particular facet of its simplicity is its on-shell constructibility $[3,4]$ : the use of (four-dimensional) unitarity cuts to construct amplitudes [5-8] finds its apotheosis in this theory, and this has led to many fruitful insights, but there are still outstanding questions about what broader statements about scattering amplitudes in more general quantum field theories can be induced from these successes.

Although QCD amplitudes remain a few steps behind in comparison, a firm foothold has been gained at two-loop order for five-gluon scattering [9-11] thanks to finite field numerical reconstruction of numerators [12] on $D$-dimensional unitarity cuts; these results have in turn been processed into more manageable functions [13-15]. A particularly manageable example at two loops is the all-plus helicity amplitude (AP amplitude for short). Due to its simpler analytic structure, particularly when arranged to separate off the infrared (IR) divergence [16], the amplitude can in fact be directly computed with one-loop methods $[17,18]$. Recent work on its subleading-colour structure $[19,20]$ has led to proofs of conformal invariance at one loop [21], and an all-multiplicity (all- $n$ ) form of a partial amplitude [22], the first such example. 
In 1996, Bern, Dixon, Dunbar and Kosower (BDDK) [23] conjectured a relation between the simplest one-loop gluon amplitudes in these two theories. When computed in $D=4-2 \epsilon$ dimensions in dimensional regularisation, they conjectured that for arbitrary multiplicity $n$,

$$
A_{n}^{\mathrm{QCD}}\left(1^{+}, 2^{+}, \ldots, n^{+}\right)=-2 \epsilon(1-\epsilon)(4 \pi)^{2}\left[\frac{A_{n}^{\mathcal{N}=4}\left(1^{+}, \ldots, i^{-}, \ldots, j^{-}, \ldots, n^{+}\right)}{\langle i j\rangle^{4}}\right]_{\epsilon \rightarrow \epsilon-2},
$$

where the change in $\epsilon$ on the right-hand-side corresponds to a "dimension shift" $4-2 \epsilon \rightarrow$ $8-2 \epsilon$. While the amplitudes on each side were by then well known up to $\mathcal{O}(\epsilon)$, there has been little motivation to develop methods to probe higher orders in $\epsilon$, and so it has been difficult to test the validity of the conjecture. A further motivation to do so comes from recent interest in properties of amplitudes viewed in terms of their expansions in multiple polylogarithms. It would be interesting to know whether these most accessible amplitudes, which are highly constrained by their myriad symmetries, are well behaved and easily predicted when probed at higher orders in $\epsilon$.

Both the all-plus and the MHV amplitudes have close connections with integrability and string theory. At one loop, the AP amplitude has been explicitly shown to be equivalent to the one-loop amplitude in self-dual Yang-Mills theory: the latter was built from the Lagrangian by Cangemi [24], as well as by Chalmers and Siegel [25] which built on previous work by Bardeen [26], who computed four and five-point amplitudes directly from all-plus tree level amplitudes with two off-shell legs, which is very close to the analysis we carry out in section 2 to all multiplicity.

Moreover Bardeen proposed that the non-vanishing of the AP amplitude at loop level was rooted in an anomaly associated with the currents in the self-dual sector; recent work along these lines explores these elemental notions in a manner complementary to the "amplitudes"-based techniques presented here [27]. Self-dual Yang-Mills has historically been a theory of interest as it is classically integrable (see [28] and references therein), and can also be obtained from the compactification of $\mathcal{N}=2$ strings in $2+2$ spacetime dimensions [29].

The technique used by BDDK in [23] for computing the all-epsilon structure of $\mathcal{N}=4$ MHV amplitude for $n=5,6$ built upon the computation of the four-point string amplitude by Green, Schwarz and Brink [30], by taking the field-theory limit of the 10-dimensional open superstring to compute the amplitude in $D=4-2 \epsilon$. Systematic rules which generalise a string-based computation of field-theory amplitudes were developed by Bern and Kosower [31], and it was shown that they can also be directly derived from the Schwinger worldline formalism as derived by Bern and Dunbar [32]. As a computational alternative to Feynman diagrams worldline approaches maintain significant interest [33, 34] providing a context to study asymptotic states in scattering problems [35], and for computing gravitational-radiation contributions to black-hole scattering [36].

Statements concerning the relationship between $\mathcal{N}=4 \mathrm{MHV}$ and AP amplitudes can also be found in the literature. Schabinger [37] has pointed out that dimension-shifted oneloop $\mathcal{N}=4$ amplitudes occur in the tree-level $\mathcal{O}\left(\alpha^{\prime 2}\right)$ string computations of Stieberger and 
Taylor [38], and that as both amplitudes are dominated by cyclic structure and $F^{4}$ terms, they are so constrained that they must coincide. This provides a strong argument for the validity of equation (1.1), and indeed the arguments we make in section 2 confirm this.

\subsection{Overview}

In supersymmetric Yang-Mills theories, the all-plus (and indeed the single-minus) gluon amplitudes can be shown to vanish to all orders in perturbation theory ${ }^{1}$ thanks to supersymmetric (SUSY) Ward identities [39],

$$
A_{n}^{\mathrm{SUSY}}\left(1^{ \pm}, 2^{+}, \ldots, n^{+}\right)=0 .
$$

At one-loop order we can decompose the gluonic ([1]), fermionic $([1 / 2])$ contributions to the loop content into terms which can be expressed entirely in terms of amplitudes in supersymmetric theories, and an independent $(\mathcal{N}=0)$ part which corresponds to the contribution from a complex scalar [40]

$$
\begin{aligned}
& A^{[0]}=\mathcal{S}, \\
& A^{\left[\frac{1}{2}\right]}=-2 \mathcal{S}+\mathcal{F}, \\
& A^{[1]}=2 \mathcal{S}+\mathcal{G} .
\end{aligned}
$$

Here $A^{[0]}$ denotes the contribution from a real scalar: as the scalar and anti-scalar contributions are equal, the complex case is retrieved by simply doubling this term. The expressions (1.5) can be seen emerge from inverting the decompostion of the supersymmetric multiplets ${ }^{2}$

$$
\begin{aligned}
A^{\mathcal{N}=4} & =A^{[1]}+4 A^{\left[\frac{1}{2}\right]}+6 A^{[0]} \\
& =\mathcal{G}+4 \mathcal{F} \\
A^{\mathcal{N}=1} & =A^{[1]}+A^{\left[\frac{1}{2}\right]} \\
& =\mathcal{G}+\mathcal{F} .
\end{aligned}
$$

From the vanishing property (1.2) we conclude that

$$
A_{n}^{[1]}(++\cdots+)=2 A^{[0]}=2 \mathcal{S} .
$$

A major simplification is thus made manifest: the AP amplitude is equivalent to having just two real scalars circulating around the loop. This makes its computation using massive cuts particularly tractable [42]. It is thus puzzling that in this context the $\mathcal{N}=4$ MHV amplitude seems to have a structure almost entirely converse to the all-plus in that equations (1.9) and (1.7) have mutually disjoint content.

\footnotetext{
${ }^{1}$ As the tree level amplitudes coincide in SUSY and in QCD, this is also a statement about massless QCD at leading order.

${ }^{2}$ They also emerge naturally from implementing the Bern-Kosower rules in the string-based formalism $[31,32,41]$.
} 
The conjecture (1.1) was verified by BDDK up to six points, with the explicit expressions for the QCD amplitudes computed to be

$$
\begin{aligned}
& A_{4}^{\mathrm{AP}} \equiv A_{4}^{\mathrm{QCD}}\left(1^{+}, 2^{+}, 3^{+}, 4^{+}\right)=\frac{-2 \epsilon(1-\epsilon)}{\langle 12 \cdots 41\rangle} s_{12} s_{23} I_{4}^{8-2 \epsilon} \\
& A_{5}^{\mathrm{AP}}=\frac{-\epsilon(1-\epsilon)}{2\langle 12 \cdots 51\rangle}\left[\sum_{j=1}^{n} s_{j+1, j+2} s_{j+2, j+3} I_{4}^{8-2 \epsilon,(j)}+(D-4) \operatorname{tr}{ }_{5}(1234) I_{5}^{10-2 \epsilon}\right], \\
& A_{6}^{\mathrm{AP}}=\frac{-\epsilon(1-\epsilon)}{2\langle 12 \cdots 61\rangle}\left[-\sum_{1<j_{1}<j_{2} \leq n}^{n} \operatorname{tr}\left(\left(j_{1}+1\right) q_{j_{1}+1, j_{2}+1}\left(j_{2}+1\right) q_{j_{2}+1, j_{1}+1}\right) I_{4}^{8-2 \epsilon,\left(j_{1}, j_{2}\right)}\right. \\
&\left.+(4-2 \epsilon)\left(\sum_{j=1}^{n} \operatorname{tr}_{5}(j+1, j+2, j+3, j+4) I_{5}^{10-2 \epsilon,(j)}+\operatorname{tr}(123456) I_{6}^{10-2 \epsilon}\right)\right],
\end{aligned}
$$

and the $\mathcal{N}=4 \mathrm{MHV}$ amplitudes can be obtained from (1.1). Here we use the variables $q_{r s}$ to denote sums of consecutive momenta $q_{r s}=\sum_{i=r}^{s-1} p_{i}$. The descendant integral notation $\left(j_{1}, j_{2}\right)$ denotes the shrinking of the propagators leading into the $j_{1}$ th and $j_{2}$ th leg of the maximal $n$-gon. We use the usual notation for two-particle Mandelstam invariants $s_{i j}=\left(p_{i}+p_{j}\right)^{2}$, and

$$
\begin{aligned}
\operatorname{tr}(a b \cdots) & =\operatorname{tr}\left(\not p_{a} \not p_{b} \cdots\right)=\operatorname{tr}_{+}(a b \cdots)+\operatorname{tr}_{-}(a b \cdots), \\
\operatorname{tr}_{5}(a b \cdots) & =\operatorname{tr}\left(\gamma_{5} \not p_{a} \not p_{b} \cdots\right)=\operatorname{tr}_{+}(a b \cdots)-\operatorname{tr}_{-}(a b \cdots),
\end{aligned}
$$

where $\operatorname{tr}_{ \pm}$involve the usual chiral projectors, and can be expressed in terms of spinorhelicity if one of the momenta is null. For example, if $p_{a}^{2}=0$ then

$$
\begin{aligned}
& \operatorname{tr}_{+}(a b \cdots)=\frac{1}{2} \operatorname{tr}\left(\left(1+\gamma_{5}\right) \not p_{a} \not p_{b} \cdots\right)=[a|b \cdots| a\rangle, \\
& \operatorname{tr}_{-}(a b \cdots)=\frac{1}{2} \operatorname{tr}\left(\left(1-\gamma_{5}\right) \not p_{a} \not p_{b} \cdots\right)=\langle a|b \cdots| a] .
\end{aligned}
$$

Although the study of scattering amplitudes has advanced significantly since this conjecture was first proposed, an explicit proof has not been presented until now. The allmultiplicity expressions for both the MHV in $\mathcal{N}=4$ and the AP amplitude were already known to leading order in $\epsilon$ at the time of the conjecture [23]. We review these results in a more contemporary framework.

If one is content with truncating terms $\sim \mathcal{O}(\epsilon)$, a one-loop amplitude that is dimensionally regulated in $4-2 \epsilon$ dimensions can be expressed in terms of a basis of box, triangle and bubble integrals with algebraic functions as coefficients, and additional purely rational terms,

$$
A_{n}=\mathbf{d}_{4} \cdot \mathbf{I}_{4}^{D=4-2 \epsilon}+\mathbf{d}_{3} \cdot \mathbf{I}_{3}^{D=4-2 \epsilon}+\mathbf{d}_{2} \cdot \mathbf{I}_{2}^{D=4-2 \epsilon}+d_{R}+\mathcal{O}(\epsilon),
$$

where $\mathbf{d}_{m} \cdot \mathbf{I}_{m}$ are dot products weighting the $m$-point scalar integrals, $I_{m}^{\left[i_{1}, \ldots, i_{m}\right]}[1]$ with algebraic functions of kinematic variables. The integral functions are defined as

$$
I_{m}^{D ;\left[i_{1}, \ldots, i_{m}\right]}[1]=i(-1)^{n+1}(4 \pi)^{2-\epsilon} e^{\gamma_{E} \epsilon} \int \frac{d^{D} \ell}{(2 \pi)^{D}} \frac{1}{\ell^{2}\left(\ell-q_{i_{1} i_{2}}\right)^{2} \cdots\left(\ell-q_{i_{1} i_{m}}\right)^{2}} .
$$


In general we denote the propagator just before the $k$ th leg $\ell_{i_{k}}$ so in equation (1.17) $\ell \equiv \ell_{i_{1}}$. The normalisation is set such that after the loop integration (but before the Feynman parameter integration) shift and reduction identities [43, 44] are simplified, consistent with [45]. We suppress the term $+i 0$ in each propagator. From here on the absence of a $D$ superscript on $I_{m}$ implies $D=4-2 \epsilon$. The argument in the square brackets, indicates possible numerator terms introduced: these could be loop momenta or Feynman parameters and thus apply at different stages of the loop integration depending on context; if no brackets are present the numerator is understood to be 1 and we speak of scalar integrals, as in the formula above.

The term $d_{R}$ in the basis (1.16) is a rational function of spinor-brackets and momentum variables only. One manifestation of the simplicity of amplitudes in $\mathcal{N}=4 \mathrm{SYM}$ at one-loop is the fact that

$$
\mathbf{d}_{i}^{\mathcal{N}}=4=\mathbf{0}, \quad \text { for } i \in\{R, 2,3\} ;
$$

while for the all-plus amplitude, the lack of four-dimensional cuts implies that

$$
\mathbf{d}_{i}^{A P}=\mathbf{0}, \quad \text { for } i \in\{2,3,4\} .
$$

We adopt the Grassmann delta-function $\delta^{(8)}\left(|i\rangle \eta_{i A}\right)$ notation [46], which bundles together the states in the supermultiplet as related by supersymmetric (SUSY) Ward identities. Negative helicity gluon states are extracted by applying the usual functional derivatives $\frac{\delta^{4}}{\delta^{4} \eta_{i}}$; in practice this amounts simply to partial derivatives and then setting all $\eta$ to 0 . This allows us to deal with the cyclically invariant MHV superamplitude, and project out gluon amplitude on the r.h.s. of equation (1.1) with

$$
A_{n}^{\mathcal{N}=4}\left(1^{+}, \ldots, i^{-}, \ldots, j^{-}, \ldots, n^{+}\right)=\frac{\delta^{4}}{\delta \eta_{i}^{4}} \frac{\delta^{4}}{\delta \eta_{j}^{4}}\left[A_{n}^{\mathrm{MHV}}\right] .
$$

In this broader framework, two-particle unitarity cuts depicted on the left of figure 1, can be expressed as a product of two MHV superamplitudes. Considering, for example, the $q_{1 r}^{2}$-channel cut,

$$
\begin{aligned}
\left.A_{n}^{\mathrm{MHV}}\right|_{q_{1 r} \text { cut }} & =\int d^{4} \eta_{\ell_{1}} d^{4} \eta_{\ell_{r}} \frac{\delta^{(8)}(L)}{\left\langle\ell_{1} 1\right\rangle\langle 12\rangle \cdots\left\langle(r-1) \ell_{r}\right\rangle\left\langle\ell_{r} \ell_{1}\right\rangle} \times \frac{\delta^{(8)}(R)}{\left\langle\ell_{r} r\right\rangle \cdots\left\langle n \ell_{1}\right\rangle\left\langle\ell_{1} \ell_{r}\right\rangle}, \\
L & \equiv|i\rangle \eta_{i A}, i \in\left\{-\ell_{1}, 1, \ldots, r-1, \ell_{r}\right\} \\
R & \equiv|i\rangle \eta_{i A}, i \in\left\{-\ell_{1}, 1, \ldots, r-1, \ell_{r}\right\} .
\end{aligned}
$$

The left-hand delta function can be made independent of the integral variables on the support of the right-hand one, by carrying out the Grassman integration

$$
\begin{aligned}
\left.A_{n}^{\mathrm{MHV}}\right|_{q_{1 r} \text { cut }}= & \frac{\delta^{(8)}(L+R)}{\left\langle\ell_{1} 1\right\rangle\langle 12\rangle \cdots\left\langle(r-1) \ell_{r}\right\rangle} \times \frac{\left\langle\ell_{1} \ell_{r}\right\rangle^{2}}{\left\langle\ell_{r} r\right\rangle \cdots\left\langle n \ell_{1}\right\rangle} \\
= & \frac{\delta^{(8)}\left(|i\rangle \eta_{i A}\right)}{2\langle 12 \ldots n 1\rangle}\left[\frac{\operatorname{tr}\left(1 q_{2(r-1)}(r-1) q_{r 1}\right)}{\ell_{2}^{2} \ell_{r-1}^{2}}+\frac{\operatorname{tr}\left(n q_{1 r}(r) q_{(r+1) n}\right)}{\ell_{n}^{2} \ell_{r+1}^{2}}\right], \\
& i \in\{1,2, \ldots, n\} .
\end{aligned}
$$

We are then left only with box contributions from four-dimensional cuts. 

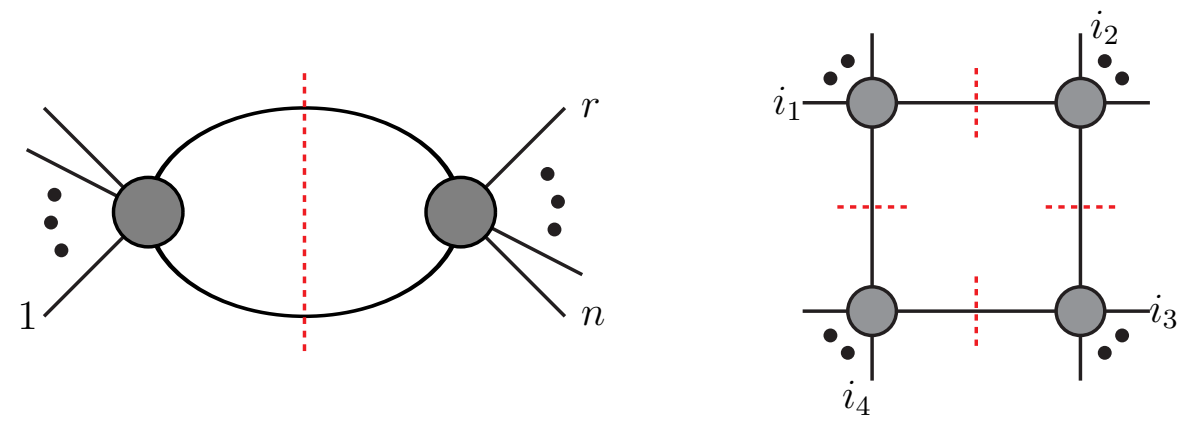

Figure 1. Old-fashioned and generalised unitarity: the two-particle cut on the left are a consistency condition on the final amplitude, which can be used to constrain its form; the generalised fourparticle cut on the right allows the direct computation of the box coefficient $d_{4}^{\left[i_{1}, i_{2}, i_{3}, i_{4}\right]}$.

We can more conveniently use generalised unitarity [7] to compute $\mathbf{d}_{4}$. Each box coefficient can be computed by imposing the four dimensional on-shell conditions

$$
\ell_{i_{k}}^{2}=\left(\ell_{1}-q_{1 i_{k}}\right)^{2}=0, \quad i \in 1,2,3,4
$$

resulting in the product of amplitudes on the generalised cut depicted on the right of figure 1. The coefficient should be averaged over the two solutions of the constraints in equation (1.23).

$$
\begin{aligned}
d_{4}^{\left[i_{1}, i_{2}, i_{3}, i_{4}\right]}=\frac{1}{2} \sum_{l_{ \pm}} A^{\text {tree }}\left(-\ell_{i_{1}}, i_{1}, \ldots, i_{2}-1, \ell_{i_{2}}\right) \times A^{\text {tree }}\left(-\ell_{i_{2}}, i_{2}, \ldots, i_{3}-1, \ell_{i_{3}}\right) \\
\\
\quad \times A^{\text {tree }}\left(-\ell_{i_{3}}, i_{3}, \ldots, i_{4}-1, \ell_{i_{4}}\right) \times A^{\text {tree }}\left(-\ell_{i_{4}}, i_{4}, \ldots, i_{1}-1, \ell_{i_{1}}\right) .
\end{aligned}
$$

The only cut configuration with non-vanishing amplitudes composing it involves opposite facing 3-point amplitudes, which upon evaluation gives

$$
d_{4}^{\left[i_{1}, i_{1}+1, i_{3}, i_{3}+1\right]}=\frac{\delta^{(8)}\left(|i\rangle \eta_{i A}\right)}{\langle 12 \ldots n 1\rangle} \times \operatorname{tr}\left(i_{1} q_{i_{1}+1, i_{3}} i_{3} q_{i_{3}+1, i_{1}}\right) .
$$

Thus the amplitude is

$$
A_{n}^{\mathrm{MHV}}=\frac{1}{4} \frac{\delta^{(8)}\left(|i\rangle \eta_{i A}\right)}{\langle 12 \ldots n 1\rangle} \sum_{i_{1}, i_{3}=1}^{n} \operatorname{tr}\left(i_{1} q_{i_{1}+1, i_{3}} i_{3} q_{i_{3}+1, i_{1}}\right) I_{4}^{\left[i_{1}, i_{1}+1, i_{3}, i_{1}+1\right]}+\mathcal{O}(\epsilon),
$$

where $I_{4}$ is in $4-2 \epsilon$ dimensions. The terms $\mathcal{O}(\epsilon)$ are (implicitly) determined in sections 2 and 3.

The AP amplitude manifests a structure which is somewhat converse; its four-dimensional unitarity cuts vanish: any on-shell QCD amplitude inserted into the cut diagrams of figure 1 with all-plus helicity external legs vanishes. It is, however, highly constrained and as such the all- $n$ form was determined ad hoc by symmetry principles and limiting behaviour $[47,48]$.

$$
A_{n}^{\mathrm{AP}}=\sum_{1 \leq i_{1}<i_{2}<i_{3}<i_{4} \leq n} \frac{\operatorname{tr}_{-}\left(i_{1} i_{2} i_{3} i_{4}\right)}{\langle 12 \ldots n 1\rangle}+\mathcal{O}(\epsilon)
$$


Equation (1.27) can be recovered from equations (1.10), (1.11), and (1.12) by noting that

$$
\begin{aligned}
& \epsilon(1-\epsilon) I_{4}=\frac{1}{6}+\mathcal{O}(\epsilon), \\
& \epsilon(1-\epsilon) I_{5}=\frac{1}{24}+\mathcal{O}(\epsilon) .
\end{aligned}
$$

The two sides of the conjecture (1.1) were computed in different ways by BDDK: the all-epsilon structure of MHV amplitude was computed using the string-based formalism whereas AP amplitude, was computed with a prototypical version of $D$-dimensional unitarity. This is effectively equivalent to taking unitarity cuts with massive on-shell states, specifically applying the techniques of Bern and Morgan [49] to compute the expressions in equations (1.10), (1.11) and (1.12); a technique made especially simple thanks to the equivalence in equation (1.9).

Moreover, as BDDK highlight, the D-dimensional unitarity technique provides the full structure of the amplitude and can thus be made use of to prove the conjecture. An alternative statement to (1.1) in terms of cuts is thus

$$
\left.A_{n}^{\mathrm{AP}}\right|_{q_{r s} \text { cut }} ^{\mu^{2} \neq 0}=\left.A^{\mathcal{N}=4}\left(1^{+}, 2^{+}, \ldots, i^{-}, \ldots, j^{-}, \ldots, n^{+}\right)\left[\frac{2 \mu^{4}}{\langle i j\rangle^{4}}\right]\right|_{q_{r s} \text { cut }} ^{\mu^{2} \neq 0} ;
$$

for any $q_{r s}^{2}$ channel. Here $\mu^{2}$ is a mass-like parameter associated with the $(-2 \epsilon)$-dimensional component of the loop integral. The statement (1.29) is proved in section 2 thanks to massive spinor-helicity formalisms [50, 51] and insights into supersymmetric Ward identities [52] for Coulomb-branch amplitudes [53-55] in particular an axial-gauge choice, which is equivalent to a choice of basis with which to define the intermediate states in the massive unitarity cut.

In section 3 an ansatz is presented for the all- $n$ all- $\epsilon$ form for the AP (and thus through the dimension shift in equation (1.1) the $\mathcal{N}=4 \mathrm{MHV}$ ) amplitude through the computation of generalised $D$-dimensional cuts, depicted in figure 2 . This method is directly analogous to the one first introduced for four-dimensional cuts in [7]; it produces a compact result thanks to the simple form of a solution to the on-shell conditions for a given pentagon, $I_{5}^{\left[i_{1}, i_{2}, i_{3}, i_{4}, i_{5}\right]}$

$$
\ell_{i_{1}}^{\nu}=-\frac{\operatorname{tr}_{5}\left(q_{i_{1} i_{2}} q_{i_{2} i_{3}} q_{i_{3} i_{4}} q_{i_{4} i_{5}} \gamma^{\nu}\right)}{2 \operatorname{tr}_{5}\left(q_{i_{1} i_{2}} q_{i_{2} i_{3}} q_{i_{3} i_{4}}\right)} .
$$

We then conclude with some observations and outlook.

\section{Proof of the conjecture}

As stated in the original discussion in [23], an equivalent statement to the conjecture (1.1) is that the $D$-dimensional cuts match:

$$
\left.A^{A P}\right|_{q_{r s} \text { cut }} ^{\mu^{2} \neq 0}=A^{\mathcal{N}=4}\left(++\cdots i^{-} \cdots j^{-} \cdots++\left.\left[\frac{2 \mu^{4}}{\langle i j\rangle^{4}}\right]\right|_{q_{r s} \text { cut }} ^{\mu^{2} \neq 0} .\right.
$$

It is in showing this relationship that we prove this conjecture here. 

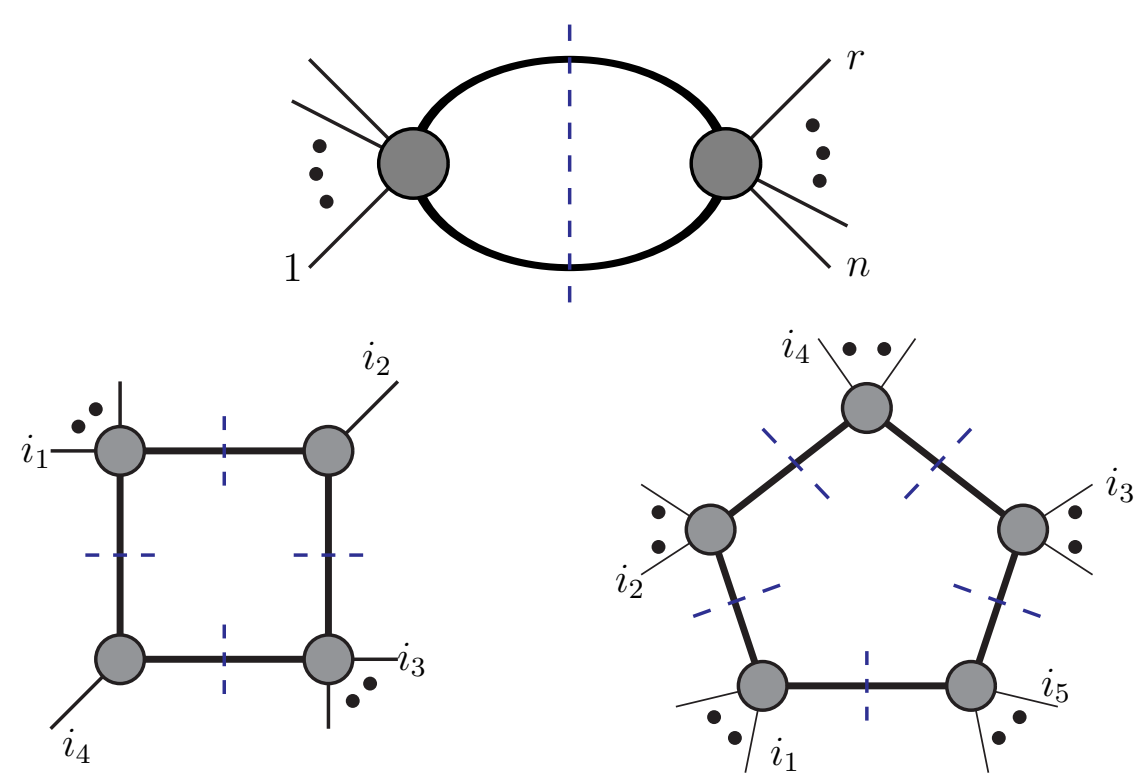

Figure 2. Top: the $D$-dimensional cuts capture the full amplitude, it is in this context that the BDDK conjecture is proved in section 2.

Bottom: generalised $D$-dimensional cuts can be used to compute the functional form of the all-plus, and thus the MHV through the relation (1.1).

\section{$2.1 \quad$ Unitarity cuts}

It has long been known that unitarity cuts provide tight constraints on the functional form of amplitudes [56] and can be used as a tool to compute them [6, 57]. A technique emblematic of contemporary on-shell methods for computing scattering amplitudes, generalised unitarity $[6,7,57-61]$, is able to fully fix box, triangle and bubble coefficients in supersymmetric theories at leading order in epsilon. However, rational terms, in particular those that compose the all-plus and single-minus amplitudes, are non-vanishing in non-supersymmetric theories; supplementary approaches [18, 42, 62-64] are needed to overcome these limitations.

A more complete unitarity technique known as " $D$-dimensional" unitarity $[42,65-67]$ extends the conventional version, but considers additional singularities of the integrand which capture the full singular structure of the amplitude; these extra singularities can be counted when the "on-shell" states of the unitarity cut are considered to be $D=4-2 \epsilon$ dimensional.

After formally splitting the $D$-dimensional loop momentum $\ell$ into a four-dimensional component and an orthogonal ( $-2 \epsilon)$-dimensional one,

$$
\ell=l^{\mu}+\ell^{[-2 \epsilon]}
$$

the cut condition becomes

$$
\ell^{2}=l^{2}-\mu^{2}=0
$$


where we have defined

$$
\mu^{2} \equiv\left(\ell^{[-2 \epsilon]}\right)^{2}
$$

The integral measure can then be transformed according to

$$
\begin{aligned}
d^{4-2 \epsilon} \ell & =-\frac{d \mu^{2}}{\left(-\mu^{2}\right)^{1+\epsilon}} d^{4} l \\
& =\frac{d \mu^{2}}{\mu^{2}} d^{4} l+\mathcal{O}(\epsilon) .
\end{aligned}
$$

The $\mu^{2}=0$ pole captures the four-dimensional structure, whereas the singular locus for the combination of all cut conditions

$$
\ell_{i}^{2}=l_{i}^{2}-\mu^{2}=0
$$

encodes all-epsilon properties with coefficients characterised by the "off-shell structure" of the tree-level theory. Thus $\mu^{2}$ can be considered as an extra parameter subject to the cut constraints, and is ultimately the mass term in the tree-level amplitudes which are used to fix the integral coefficients.

As with four-dimensional unitarity, a set of $k$ massive-cut conditions singles out a codimension- $k$ singular locus in the domain of integration; the extra integral parameter $\mu^{2}$ puts the dimension of this domain, and thus the number of constraints on the maximal cut, at $k=5$ as opposed to $k=4$ in the massless (or $\mu^{2}=0$ ) case. Thus for a $k$-line cut, $k-5$ free parameters are unfixed by the constraints. The coefficients can be determined from these cut expressions through taking careful limits depending on the residues coming from poles in the unfixed parameters $[42,59]$. How this is done is contingent on a choice of integral basis: both the AP and MHV amplitudes are free of bubble and triangle contributions, so just the $\mu^{2}$ degree of freedom need be considered to capture box coefficients. The discussion of what basis to use in computation of the amplitudes is carried out in section 3 .

Four-dimensional cuts constrain an amplitude by requiring that any two-particle cuts reproduce a product of tree-level amplitudes

$$
\left.A_{n}\right|_{q_{r s} \text { cut }} ^{\mu^{2}=0}=\sum_{h_{s}, h_{r}} A_{r+2}^{\text {tree }}\left(-l_{r}^{h_{r}}, r, r+1, \ldots, s-1, l_{s}^{-h_{s}}\right) \times A_{n-r+2}^{\text {tree }}\left(-l_{s}^{-h_{s}}, s, s+1, \ldots, r-1, l_{r}^{h_{r}}\right) .
$$

This can be fit into a broader framework by noting that the eigenstates of the little group for massless particles, U(1), are represented by the sum over helicities. The helicity "weight" which induces a helicity weighting (or complex phase) for each particle cancels across the cut; this is how the cut can be viewed as a U(1) contraction. In theories with rational terms, cuts of the form (2.7) are incomplete, as a consequence of the intermediate states living in $D=4-2 \epsilon$ dimensions. Taking a $D$-dimensional unitarity requires a concrete scheme for defining these states crossing the cut. This has previously been done using sixdimensional spinor helicity [68-70], but here we propose simply using off-shell/equal-mass amplitudes. 
Equation (2.5) provides a natural motivation to use massive amplitudes. ${ }^{3}$ An heuristic justification for this is the fact renormalisation and resolution of infrared singularities always introduces an external scale, and thus off-shell expressions of the classical theory will be needed to fully define a loop amplitude. The massive spinor-helicity formalism of Arkani-Hamed, Huang and Huang [51] (AHH) provides a contemporary framework for combining these expressions.

In the AHH formalism, a two-particle unitarity cut consists of a sum over several inner products each carried out over the particles crossing the cut

$$
\left.A_{n}\right|_{q_{r s} \text { cut }} ^{\mu^{2} \neq 0}=\sum_{\text {particles }}\left\langle A_{L}^{\text {tree }}\left(-\boldsymbol{l}_{r}^{\sigma_{r}}, r, r+1, \ldots, s-1, \boldsymbol{l}_{s}^{\sigma_{s}}\right), A_{R}^{\text {tree }}\left(-l_{r}^{\sigma_{s}}, s, \ldots, r-1, \boldsymbol{l}_{r}^{\sigma_{r}}\right)\right\rangle,
$$

where the boldface notation indicates suppressed, symmetrised $\mathrm{SU}(2)$ indices, the little group of massive particles in $3+1$ dimensions. The inner product contracts these indices, and the contraction has a clear interpretation in terms of the eigenstates of the $\mathrm{SU}(2)$ little group of the particles of spin $\sigma$ which "cross" the cut. The AHH formalism thus represents this product as rank- $\left(2 \sigma_{s}+2 \sigma_{r}\right)$ tensors of $\mathrm{SU}(2)$ contracted with two-dimensional LeviCivita symbols $\epsilon_{\alpha_{j}^{r} \beta_{j}^{r}}$ and $\epsilon_{\alpha_{k}^{s} \beta_{k}^{s}}$,

$$
\left.A_{n}\right|_{q_{r s} \text { cut }} ^{\mu^{2} \neq 0}=\sum_{\text {particles }} A_{L}^{\alpha_{1}^{r} \ldots \alpha_{2 \sigma_{r}}^{r} ; \alpha_{1}^{s} \ldots \alpha_{2 \sigma_{s}}^{s}} \prod_{j=1}^{2 \sigma_{r}}\left[\epsilon_{\alpha_{j}^{r} \beta_{j}^{r}}\right] \prod_{k=1}^{2 \sigma_{s}}\left[\epsilon_{\alpha_{k}^{s} \beta_{k}^{s}}\right] A_{R}^{\beta_{1}^{s} \ldots \beta_{2 \sigma_{s}}^{s} ; \beta_{1}^{r} \ldots \beta_{2 \sigma_{r}}^{r}} .
$$

There is also a constraint on the expressions which feed into the cut expression (2.8): the quantum states in a given channel must be consistently defined, i.e. defined in the same frame of reference. In practice this is simply done by fixing an axial gauge vector, ${ }^{4}$ which we term $p_{\chi}$, against which to define helicities, or spin states of massive particles.

To use these tools to prove the formula (2.1) we require the tree-level expressions.

\subsection{Tree-level off-shell amplitudes}

The all-multiplicity tree-level expressions that need to be fed into equation (2.8) have been known for a long time and exist in multiple forms throughout the literature [54, 72 75], mostly in the older massive spinor-helicity formalism of Dittmaier [50]. As with the tree amplitudes of all-massless particles, compact expressions for massive amplitudes are neatly related to each other through SUSY Ward identities [52, 76]. These can be bundled together in a super-amplitude like the massless-MHV case, with a clear interpretation in terms of taking $\mathcal{N}=4$ away from the origin of the moduli space [53,54], where the origin corresponds to the massless theory. ${ }^{5}$ The result is a super-amplitude in what is termed

\footnotetext{
${ }^{3}$ The fact that Pauli-Villars regularisation [71] functions by introducing masses also supports this.

${ }^{4}$ This is most often denoted as $q$ in the literature. It is equivalent to fixing a Lorentz frame and thus is also referred to as a " $q$-frame" in e.g. [54]. Note also that choosing $p_{\chi}$ to be complex is also an option.

${ }^{5}$ The Coulomb branch can also be obtained by compactifying the massless $\mathcal{N}=(1,1)$ maximal theory in 6 dimensions [68, 69].
} 
"MHV band" [53]. The MHV-band superamplitude differs from the massless MHV superamplitude in that the delta functions are not homogeneous in the $\eta$ variables which carry the helicity weight. We discuss how to interpolate between the two formalisms and collect the necessary expressions here.

The AHH massive spinor-helicity formalism [51] deployed here extends the compactness of conventional spinor helicity by defining a given amplitude with massive external particles 1 and $n$, of spin $\sigma_{1}$ and $\sigma_{n}$ respectively, as a rank $2 \sigma_{1}+2 \sigma_{n}$ tensor of $\mathrm{SU}(2)$, the little group for massive particles:

$$
A_{n}^{\text {tree }}\left(\mathbf{1}^{\sigma_{1}}, 2^{h_{2}}, \ldots,(n-1)^{h_{n-1}}, \mathbf{n}^{\sigma_{n}}\right) .
$$

In practice, to compute a cut, we will need to choose a basis and express the tree-level amplitudes on the right hand side of equation (2.9) in the form

$$
A_{n}^{\text {tree }}\left(\mathbf{1}^{\sigma_{1}}, 2^{h_{2}}, \ldots, \overline{\mathbf{n}}^{\sigma_{n}}\right)=\sum_{h_{1}, h_{n}} A_{n}^{h_{1} h_{n}} \boldsymbol{\zeta}^{h_{1}} \boldsymbol{\zeta}^{h_{2}}
$$

here, on the l.h.s. $\sigma_{i}\left(h_{i}\right)$ is the spin (helicity) of the massive (massless) particle; on the r.h.s. we use indices $h_{1}, h_{n}$ to label the spin states of the massive particles in the chosen basis. The boldface variables $\zeta \equiv \prod_{k=1}^{2 \sigma} \zeta^{\alpha_{k}}$ again carry suppressed $\mathrm{SU}(2)$ tensor indices $\alpha_{k}$. Each tensor indexes the spin-states of the massive particles in a given frame of reference, or more specifically relative to a choice of axial gauge vector, which we term $p_{\chi}$. These spinning states are pseudo-orthonormal,

$$
\left\langle\boldsymbol{\zeta}^{h_{a}}, \boldsymbol{\zeta}^{-h_{b}}\right\rangle=( \pm 1)^{2 h_{a}} \delta_{a b}
$$

meaning the product in equation (2.8) can be expressed as a sum over products of complex valued functions in a given $p_{\chi}$ basis. As demonstrated by AHH in [51], the high-energy limits $\left(m^{2} \rightarrow 0\right)$ of the coefficients $A_{n}^{h_{1} h_{n}}$ are the tree amplitudes of massless particles.

AP amplitudes must satisfy the constraint in equation (2.9); thanks to all- $n$ expressions for all-plus helicity gluons with two other massive particles, the AP amplitude can be reconstructed by the product of scalar amplitudes that was exploited in [23]

$$
\begin{aligned}
\left.A_{n}^{\mathrm{AP}}\right|_{q_{r s} \text { cut }} ^{\mu^{2} \neq 0} & =2\left\langle A_{L}^{\text {tree }}\left(-\boldsymbol{l}_{r}^{0}, r, r+1, \ldots, s-1, \boldsymbol{l}_{s}^{0}\right), A_{R}^{\text {tree }}\left(-\boldsymbol{l}_{s}^{0},, s, s+1, \ldots, r-1, \boldsymbol{l}_{r}^{0}\right)\right\rangle \\
& =2 A_{L}^{\text {tree }}\left(-\boldsymbol{l}_{r}^{0}, r, r+1, \ldots, s-1, \boldsymbol{l}_{s}^{0}\right) \times A_{R}^{\text {tree }}\left(-\boldsymbol{l}_{s}^{0},, s, s+1, \ldots, r-1, \boldsymbol{l}_{r}^{0}\right),
\end{aligned}
$$

as is consistent with the string-derived equation (1.9). In the scalar case, the amplitudes are scalar functions thus reducing the inner product to conventional multiplication.

The original computations which resulted in equations (1.10)-(1.12) reconstructed integrand polynomials in the parameter $\mu^{2}$ and entailed finding a function which was consistent with it. In section 3 we will adopt a different and more direct approach, which builds on the work of Badger [42] to directly extract the coefficients of a scalar integral in a simple manner. It is the functions $A_{n}^{\text {tree }}$ needed as input for the cut constraints in equation (2.9) which 
are the focus of this section. To evaluate the cut AP amplitudes we use the all-multiplicity expressions for tree-level amplitudes of positive-helicity gluons and two equally massive scalars, first computed by Forde and Kosower through recursion relations [72]:

$$
A^{\text {tree }}\left(\mathbf{1}^{0}, 2^{+}, 3^{+}, \ldots,(n-1)^{+}, \mathbf{n}^{0}\right)=\frac{-\sum_{j=1}^{\left\lfloor\frac{n}{2}\right\rfloor-1} \kappa_{j}\left(-m^{2}\right)^{j}}{\left(s_{12}-m^{2}\right)\langle 23 \ldots(n-2)(n-1)\rangle\left(s_{n-1, n}-m^{2}\right)}
$$

with

$$
\kappa_{j}=\sum_{\left\{w_{i}\right\}_{i=1}^{j-1}}^{n-3} \frac{\left[2\left|1 q_{2 w_{1}} w_{1} q_{w_{1} w_{2}} w_{2} \cdots w_{j-1} q_{w_{j-1} w_{j}}\right| n-1\right]}{\prod_{r=1}^{j-1}\left(q_{1\left(w_{r}\right)}^{2}-m^{2}\right)\left(q_{1 w_{r}+1}^{2}-m^{2}\right)}
$$

where the sets $\left\{w_{i}\right\}$ are defined by the conditions: ${ }^{6}$

$$
w_{j}=n-1, \quad w_{0}=1, \quad w_{i}=w_{i-1}+2 .
$$

Other more compact representations of $A_{n}^{\text {tree }}\left(\mathbf{1}^{0}, 2^{+}, 3^{+}, \ldots,(n-1)^{+}, \mathbf{n}^{0}\right)$ have also been computed [54, 73-75]. However, we would like a representation that is arranged as a series in $m^{2}$ with only at worst linear dependence on the massive momenta in the numerators, which helps to insert cut solutions into amplitudes. By means of the expression (2.14) for $n=4,5,6$, BDDK were able to confirm the representations (1.10), (1.11), and (1.12) of the AP amplitudes.

The $\mathcal{N}=4$ super-multiplet is more complicated to treat in this manner. We can begin by exploring the nature of off-shell amplitudes: how does the cut (2.9) look if quarks or vector bosons were circulating in the loop? As mentioned above, projection onto a state basis of the kind expressed in equation (2.11) can be done by choosing a reference fourvector, $p_{\chi}$, relative to which we define the spinning particles. We build our basis using this reference, and interpolate between old [50] and new [51] massive spinor-helicity formalisms. This is ultimately equivalent to making a choice of axial gauge [77] and thus this choice is referred to simply as a gauge from here on.

Considering massive vector bosons $\mathbf{1}^{1}$ and $\mathbf{n}^{1}$ having opposite helicity, it is straightforward to express the equivalent of equation (2.14): SUSY Ward identities [52, 76] can be used to show that the vector boson just involves the introduction of an overall factor relative to the scalar case. The spin-one state coefficient of $\boldsymbol{\zeta}_{1}^{-} \boldsymbol{\zeta}_{n}^{+}$in the expansion of equation (2.11) is

$$
A_{n}^{-+}\left(\mathbf{1}^{1}, 2^{+}, 3^{+}, \ldots,(n-1)^{+}, \mathbf{n}^{1}\right)=\frac{\left\langle\chi \lambda_{1}\right\rangle^{2}}{\left\langle\chi \lambda_{n}\right\rangle^{2}} A_{n}^{\text {tree }}\left(\mathbf{1}^{0}, 2^{+}, 3^{+}, \ldots,(n-1)^{+}, \mathbf{n}^{0}\right)
$$

where $\langle\chi|$ is a reference spinor corresponding to the gauge choice and $\left|\lambda_{i}\right\rangle$ represents $^{7}$ the holomorphic spinor of the nullified momentum $p_{\lambda_{i}}$ defined such that

$$
p_{\lambda_{i}}=p_{i}-\frac{m^{2}}{[\chi|i| \chi\rangle} p_{\chi} .
$$

\footnotetext{
${ }^{6}$ See also [72] for more explicit representations of the sum over $\left\{w_{j}\right\}$.

${ }^{7}$ Also denoted $\left|\lambda_{i}\right\rangle \equiv\left|i^{\text {b }}\right\rangle$ in [77] or $\left|i^{\perp}\right\rangle$ in [53-55].
} 
We can extract the full rank $2 \sigma_{1}+2 \sigma_{n}$ tensor amplitude by loosening the $\chi$ constraint, and considering how component amplitudes $A_{n}^{h_{1} h_{n}}$ in a particular gauge fix the full SU(2) tensor structure of the amplitude through symmetry principles. By fixing $\langle\chi| \rightarrow\left\langle\chi_{n}\right|$ where $\chi_{n}$ is defined such that $\left\langle\chi_{n} \lambda_{n}\right\rangle=m$ we get

$$
A_{n}^{-+}\left(\mathbf{1}^{1}, 2^{+}, 3^{+}, \ldots,(n-1)^{+}, \mathbf{n}^{1}\right)=\frac{\left\langle\chi_{n} \lambda_{1}\right\rangle^{2}}{m^{2}} A_{n}^{\text {tree }}\left(\mathbf{1}^{0}, 2^{+}, 3^{+}, \ldots,(n-1)^{+}, \mathbf{n}^{0}\right) .
$$

The spinor $\chi_{n}$ is a specific choice which favours the taking of the high-energy limit of tree expressions in the $\mathrm{AHH}$ scheme $^{8}$ [51]. In general the momentum of each massive particle can be decomposed

$$
\begin{aligned}
\left(p_{i}\right)_{\dot{a} b} & =\left(p_{\lambda_{i}}-p_{\chi_{i}}\right)_{\dot{a} b}=\lambda_{\dot{a}}^{I} \bar{\lambda}_{I b}= \\
\Rightarrow \lambda_{\dot{a}}^{I} & =\lambda_{\dot{a}}\left(\zeta^{-}\right)^{I}+\chi_{\dot{a}}\left(\zeta^{+}\right)^{I}, \\
\bar{\lambda}_{b}^{I} & =\bar{\lambda}_{b}\left(\zeta^{+}\right)^{I}+\bar{\chi}_{b}\left(\zeta^{-}\right)^{I},
\end{aligned}
$$

and the spinors fixed such that $\langle\lambda \chi\rangle=[\lambda \chi]=m$. Thus from fixing the gauge $\chi \rightarrow \chi_{n}$ it is easy to induce the gauge-covariant rank 4 amplitude from the $\mathrm{SU}(2)$ symmetry by promoting the coefficient of $\boldsymbol{\zeta}_{1}^{-} \boldsymbol{\zeta}_{n}^{+}$, the gauge-dependent factor in equation (2.19) can thus be generalised:

$$
A_{n}^{\text {tree }}\left(\mathbf{1}^{1}, 2^{+}, 3^{+}, \ldots,(n-1)^{+}, \mathbf{n}^{1}\right)=\frac{\langle\mathbf{1} \mathbf{n}\rangle^{2}}{m^{2}} A_{n}^{\text {tree }}\left(\mathbf{1}^{0}, 2^{+}, 3^{+}, \ldots,(n-1)^{+}, \mathbf{n}^{0}\right) .
$$

The expression (2.21) can also be projected in the frame of the massive particles spinning in the "negative" direction relative to their motion.

$$
A_{n}^{--}\left(\mathbf{1}^{1}, 2^{+}, 3^{+}, \ldots,(n-1)^{+}, \mathbf{n}^{1}\right)=\frac{\left\langle\lambda_{1} \lambda_{n}\right\rangle^{2}}{m^{2}} A_{n}^{\text {tree }}\left(\mathbf{1}^{0}, 2^{+}, 3^{+}, \ldots,(n-1)^{+}, \mathbf{n}^{0}\right),
$$

and from this it can be seen that

$$
\lim _{m^{2} \rightarrow 0}\left[A_{n}^{--}\left(\mathbf{1}^{1}, 2^{+}, 3^{+}, \ldots,(n-1)^{+}, \mathbf{n}^{1}\right)\right]=A_{n}^{\text {tree }}\left(1^{-}, 2^{+}, 3^{+}, \ldots,(n-1)^{+}, n^{-}\right),
$$

providing a strong consistency check.

The quark version of (2.21) has also been computed [74, 75] and is simply

$$
A_{n}^{\text {tree }}\left(\mathbf{1}^{\frac{1}{2}}, 2^{+}, 3^{+}, \ldots,(n-1)^{+}, \mathbf{n}^{\frac{1}{2}}\right)=\frac{\langle\mathbf{1} \mathbf{n}\rangle}{m} A_{n}^{\text {tree }}\left(\mathbf{1}^{0}, 2^{+}, 3^{+}, \ldots,(n-1)^{+}, \mathbf{n}^{0}\right) .
$$

The simple relation between the scalar, fermion and gluon form factors in equations (2.14), (2.21) and (2.24) can be understood simply as a consequence of supersymmetric Ward identities $[52,76]$ : they are in the highly constrained "ultra-helicity-violating" (UHV) sector [53], so called because the particles spin states have one fewer negative helicity states ${ }^{9}$ than the MHV helicity configuration.

\footnotetext{
${ }^{8} \mathrm{In}[51]$ they are denoted $\eta$ however we reserve the symbol $\eta$ to denote the Grassmann variables in the superamplitude.

${ }^{9}$ The configurations of equation (2.17) would correspond to a single-minus amplitude, and indeed vanishes in the $m^{2} \rightarrow 0$ limit.
} 
The fact that the full tensor-amplitude (2.21) produces the MHV case for free is a feature of the blurring of the normally clear demarcation between helicity states in the massive/spin case, unlike the simpler massless/helicity case. As with the massless case, however, these amplitudes can all be bundled into a super-amplitude of $\mathcal{N}=4$ taken away from the origin of the moduli space on the Coulomb branch: the "MHV-band" amplitudes read thus [53]

$$
A_{\text {tree }}^{\mathrm{MHV}-\text { band }}=\frac{\left[\lambda_{n} \lambda_{1}\right]^{2} \delta_{12}^{\chi} \delta_{34}^{\chi}}{m^{2} q_{n 2}^{4}} A\left(\mathbf{1}^{0}, 2^{+}, 3^{+}, \ldots,(n-1)^{+}, \mathbf{n}^{0}\right) .
$$

The expression $\delta_{12}^{\chi} \delta_{34}^{\chi}$ is a nonhomogeneous polynomial in the helicity (or more precisely spin-state) weight carrying variables $\eta_{i a}$. Component amplitudes corresponding to different external states can be extracted from the $\delta_{12}^{\chi} \delta_{34}^{\chi}$ in an identical way to how MHV states can be extracted from $\delta^{(8)}\left(|i\rangle \eta_{i a}\right)$ using functional derivatives. As for the massive vector boson amplitude in equation (2.21), gauge covariance implies that a single class of amplitude contains multiple spin states.

The structure and construction techniques of Coulomb branch super-amplitudes is covered extensively with the Dittmaier massive-spinor-helicity formalism in references [53$55]$, and we refer the reader to these for more background. The explicit general $p_{\chi}$-gauge form of $\delta_{12}^{\chi} \delta_{34}^{\chi}$ can be found in [53], but we give an explicit form after we fixing $p_{\chi} \cdot q_{r s}=0$, which is key to the proof of the conjecture in the following section.

\subsection{Proving the conjecture from the Coulomb branch}

To prove the conjecture we consider the individual contributions of particles as expressed in equation (1.6).

In the case where purely gluonic states are crossing a cut, we consider the fully cut MHV amplitude. Examples of the helicity configurations corresponding to the cuts, and thus the tree amplitudes that compose them, are depicted in figure 3: the types of cut possible are
a. $\mathrm{AP} \times \mathrm{N}^{2} \mathrm{MHV}$
b. $\mathrm{UHV} \times \mathrm{NMHV}$
c. $\mathrm{MHV} \times \mathrm{MHV}$.

a. These cuts do not contribute as the all-plus tree amplitude vanishes in any $p_{\chi}$ gauge [52]

$$
A^{++; \mathrm{p}_{\chi}}\left(-\boldsymbol{l}_{r}^{1}, r^{+}, \ldots, \ldots,(s-1)^{+}, \boldsymbol{l}_{s}^{1}\right)=0 .
$$

b. The UHV $\times$ NMHV cut can be extracted from the MHV-band amplitude in equation $(2.25)$ in $p_{\chi} \cdot q_{r s}=0$ gauge [53]

$$
\frac{\delta_{12}^{\chi} \delta_{34}^{\chi}}{\mu^{2}\left\langle\lambda_{l_{r}} \lambda_{l_{s}}\right\rangle^{2}} A\left(-l_{r}^{0}, r^{+}, \ldots,(s-1)^{+}, \boldsymbol{l}_{s}^{0}\right)
$$




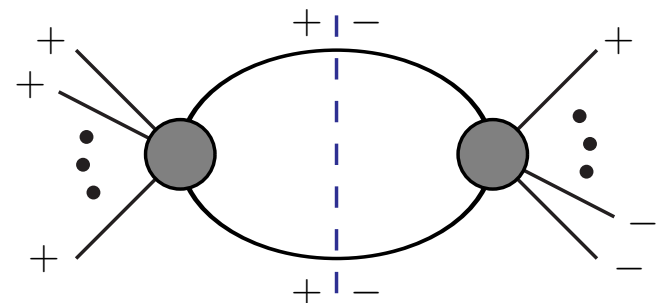

a.

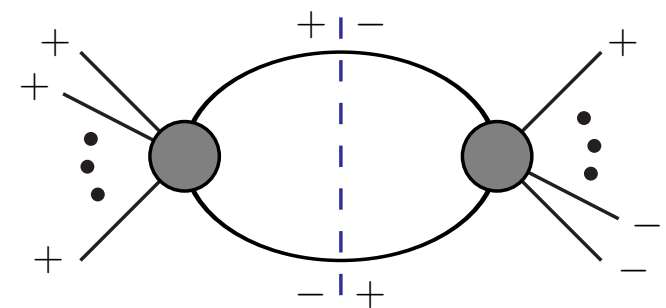

b.

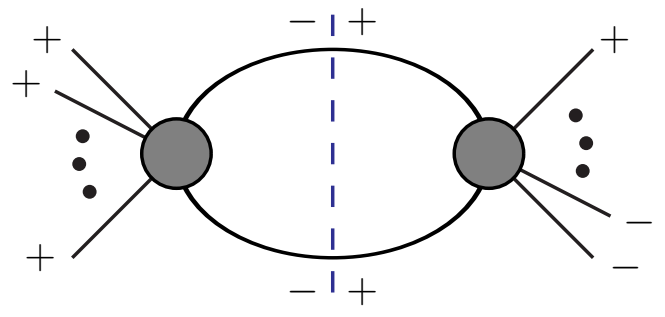

c.

Figure 3. Example helicity configurations of a. b. and c. Only c., the MHV $\times$ MHV cut survives in the full $\mathcal{N}=4$ multiplet in $q_{r s} \cdot p_{\chi}$ gauge.

with

$$
\delta_{12}^{\chi}=\left[\delta^{(4)}\left(\left\langle\lambda_{i}\right| \eta_{i a}\right)+\frac{\mu\left\langle\lambda_{l_{r}} \lambda_{l_{r}}\right\rangle}{\left\langle\chi \lambda_{l_{s}}\right\rangle\left\langle\chi \lambda_{l_{r}}\right\rangle} \delta^{(2)}\left(\left\langle\chi \lambda_{i}\right\rangle \eta_{i a}\right)\right] \times\left[1-\frac{\left[\lambda_{l_{s}} \chi\right]\left[\lambda_{l_{r}} \chi\right]}{\mu\left[\lambda_{l_{r}} \lambda_{l_{s}}\right]} \delta^{(2)}\left(\frac{\mu \eta_{i a}}{\left[\lambda_{i} \chi\right]}\right)\right]
$$

with the index $a \in\{1,2\}$ in this case, and $i \in\left\{-l_{r}, r, \ldots, s-1, l_{s}\right\} . \delta_{34}^{\chi}$ takes the same form but carries the index of the other $\mathrm{SU}(2)$ branch of the broken $\mathrm{SU}(4) R$ symmetry [53]. We can apply the functional derivative $\frac{\delta^{4}}{\delta \eta_{1}^{4}}$ to extract the UHV case: the negatively spinning term comes entirely from the second term in the first bracket giving

$$
\delta_{12}^{\chi} \delta_{34}^{\chi} \rightarrow \mu^{2}\left\langle\lambda_{r} \lambda_{s}\right\rangle^{2} \frac{\left\langle\chi \lambda_{l_{r}}\right\rangle^{2}}{\left\langle\chi \lambda_{l_{s}}\right\rangle^{2}}
$$

as is consistent with equation (2.21). The NMHV amplitude $A^{-+}\left(\boldsymbol{l}_{s}^{1}, s, \ldots, r-1, \boldsymbol{l}_{r}^{1}\right)$ comes from the term

$$
\delta_{12}^{\chi} \delta_{34}^{\chi} \rightarrow \delta^{(8)}\left(\left\langle\lambda_{i}\right| \eta_{i a}\right) \frac{\mu^{2}}{\left[\lambda_{l_{r}} \lambda_{l_{s}}\right]^{2}}\left[\frac{\left[\lambda_{l_{r}} \chi\right]^{2}}{\left[\lambda_{l_{s}} \chi\right]^{2}},\right.
$$

where in this case $i \in\{s, s+1, \ldots, r-1\}$. The gauge choice $p_{\chi} \cdot q_{r s}=0$ implies

$$
\begin{aligned}
{\left[\chi\left|l_{r}\right| \chi\right\rangle } & =\left[\chi\left|l_{s}\right| \chi\right\rangle \\
=\left[\chi \lambda_{l_{r}}\right]\left\langle\lambda_{l_{r}} \chi\right\rangle & =\left[\chi \lambda_{l_{s}}\right]\left\langle\lambda_{l_{s}} \chi\right\rangle,
\end{aligned}
$$

and thus

$$
\left[\lambda_{l_{r}} \lambda_{l_{s}}\right]\left\langle\lambda_{l_{r}} \lambda_{l_{s}}\right\rangle=q_{r s}^{2}
$$


so that the generic $\mathrm{UHV} \times \mathrm{NMHV}$ cut can be reduced to

$$
\begin{aligned}
& A_{L}^{-+}\left(-\boldsymbol{l}_{r}^{1}, r^{+}, \ldots, \ldots,(s-1)^{+}, \boldsymbol{l}_{s}^{1}\right) \times A_{R}^{+-}\left(-\boldsymbol{l}_{s}^{1}, s, \ldots, r-1, \boldsymbol{l}_{r}^{1}\right)= \\
& A_{L}\left(-\boldsymbol{l}_{r}^{0}, r^{+}, \ldots, \ldots,(s-1)^{+}, \boldsymbol{l}_{s}^{0}\right) \times \frac{\left\langle\chi \lambda_{l_{r}}\right\rangle^{2}\left[\lambda_{l_{s}} \chi\right]^{2}}{\left\langle\chi \lambda_{l_{s}}\right\rangle^{2}\left[\lambda_{l_{r}} \chi\right]^{2}} \times \frac{\delta^{(8)}\left(\left\langle\lambda_{i}\right| \eta_{i a}\right) \mu^{2}}{q_{r s}^{4}} A_{R}\left(-\boldsymbol{l}_{s}^{0}, s^{+}, \ldots,(r-1)^{+}, \boldsymbol{l}_{r}^{0}\right) .
\end{aligned}
$$

After making the identification from equation (2.25) (with corresponding arguments) and the definition in equation (2.28) then we can match

$$
\frac{\delta^{4}}{\delta^{4} \eta_{i}} \frac{\delta^{4}}{\delta^{4} \eta_{j}} A_{\text {tree }}^{\mathrm{MHV}-\text { band }}=\frac{\delta^{4}}{\delta^{4} \eta_{i}} \frac{\delta^{4}}{\delta^{4} \eta_{m}} \frac{\delta^{(8)}\left(\left\langle\lambda_{i}\right| \eta_{i a}\right) \mu^{2}}{q_{r s}^{4}} A_{R}\left(\boldsymbol{l}_{s}^{0}, s^{+}, \ldots,(r-1)^{+}, \boldsymbol{l}_{r}^{0}\right),
$$

and we identify the right-most factor in equation (2.33) as the NMHV two-massivescalar amplitude. Thus, as

$$
\frac{\left\langle\chi\left(-\lambda_{l_{r}}\right)\right\rangle\left[\lambda_{l_{r}} \chi\right]}{\left\langle\chi \lambda_{l_{s}}\right\rangle\left[\left(-\lambda_{l_{s}}\right) \chi\right]}=-1
$$

we get

$$
A_{L}^{-+} \times A_{R}^{+-}=A_{L}^{00} \times A_{R}^{00}=\left.A^{[0]}\right|_{q_{r s} \text { cut }} ^{\mu^{2} \neq 0} .
$$

From this, we identify the NMHV $\times \mathrm{UHV}$ contribution to the $q_{r s}$ gluon cut channel as that originating from $\mathcal{S}$ in the decomposition (1.5). The $A_{L}^{+-} \times A_{R}^{-+}$term is the same to make the total contribution $2 \mathcal{S}$. Similar reasoning can be used to deduce a similar result for fermions

$$
\left.A^{\left[\frac{1}{2}\right]}\right|_{\mathrm{UHV} \times \mathrm{NMHV} q_{r s} \text { cut }} ^{\mu^{2} \neq 0}=-\left.2 A^{[0]}\right|_{q_{r s} \text { cut }} ^{\mu^{2} \neq 0},
$$

and their contributions sum up to cancel with the scalar states in the $\mathcal{N}=4$ supermultiplet.

$$
\left.A_{n}^{\mathrm{MHV}}\right|_{\mathrm{UHV} \times \mathrm{NMHV} q_{r s} \text { cut }} ^{\mu^{2} \neq 0}=0 .
$$

Of course this could all be done formally using the CSW rules developed in [54, 55], but treating the particles explicitly shows how this gauge separates the structure in terms of the decomposition in equation (1.5).

c. This leaves the MHV $\times$ MHV cuts. These simply correspond to the complete unitarity cut version of equation (1.22)

$$
\begin{gathered}
\int d^{4} \eta_{l_{r}} d^{4} \eta_{l_{s}} A_{L}^{\mathrm{MHV} \text { tree }}\left(-\boldsymbol{l}_{r}^{1}, r, \ldots,(s-1), \boldsymbol{l}_{s}^{1}\right) \times A_{R}^{\mathrm{MHV} \text { tree }}\left(-\boldsymbol{l}_{r}^{1}, r, \ldots, s-1, \boldsymbol{l}_{s}^{1}\right)= \\
\int d^{4} \eta_{l_{r}} d^{4} \eta_{l_{s}} \frac{\delta^{(8)}(L)}{\mu^{2}\left\langle\lambda_{l_{s}} \lambda_{l_{r}}\right\rangle^{2}} A_{L}\left(-\boldsymbol{l}_{r}^{0}, r, \ldots,(s-1), \boldsymbol{l}_{s}^{0}\right) \times \frac{\delta^{(8)}(R)}{\mu^{2}\left\langle\lambda_{l_{s}} \lambda_{l_{r}}\right\rangle^{2}} A_{R}\left(-\boldsymbol{l}_{r}^{0}, s, \ldots, r-1, \boldsymbol{l}_{r}^{0}\right), \\
L \equiv|i\rangle \eta_{i A}, i \in\left\{\lambda_{-l_{r}}, r, \ldots, s-1, \lambda_{l_{s}}\right\} ; R \equiv|i\rangle \eta_{j A}, i \in\left\{\lambda_{l_{s}}, r, \ldots, s-1, \lambda_{-l_{r}}\right\} ;
\end{gathered}
$$


so the Grassman integration gives

$\left.A^{\mathrm{MHV}}\right|_{q_{r} \text { cut }} ^{\mu^{2} \neq 0}=\frac{\delta^{(8)}\left(\left\langle\lambda_{i}\right| \eta_{i A}\right)}{\mu^{4}} A_{L}\left(-\boldsymbol{l}_{r}^{0}, r^{+}, \ldots,(s-1)^{+}, \boldsymbol{l}_{s}^{0}\right) A_{R}\left(-\boldsymbol{l}_{s}^{1}, s^{+}, \ldots,(r-1)^{+}, \boldsymbol{l}_{r}^{1}\right)$,

equivalent to equation (2.1) upon applying the functional derivatives $\frac{\delta^{4}}{\delta \eta_{i}^{4}} \frac{\delta^{4}}{\delta \eta_{j}^{4}}$,

$$
\begin{aligned}
\left.A_{n}^{\mathcal{N}=4}\left(1^{+}, 2^{+}, \ldots, i^{-}, \ldots, j^{-}, \ldots, n^{+}\right)\right|_{q_{r s} \text { cut }} ^{\mu^{2}} & =\frac{\delta^{4}}{\delta \eta_{i}^{4}} \frac{\delta^{4}}{\delta \eta_{j}^{4}}\left[\left.A_{n}^{\mathrm{MHV}}\right|_{q_{r s} \text { cut }} ^{\mu^{2} \neq 0}\right] \\
& =\left.\frac{\langle i j\rangle^{4}}{2 \mu^{4}} A_{n}^{\mathrm{AP}}\right|_{q_{r s} \text { cut }}
\end{aligned}
$$

thus proving the conjecture (1.1).

\section{Generalised cuts and all-epsilon forms}

To compute the one-loop scattering amplitudes of the verified conjecture (1.1) we present a new generalised version of $D$-dimensional unitarity which extracts the coefficients of a fixed basis of integrals. The simplicity of one-loop amplitudes in general, and the all-plus amplitude in particular leads us to an all-multiplicity form to all orders in epsilon which, through what is now the shift identity (1.1), delivers the all-multiplicity form of the oneloop $\mathrm{MHV} \mathcal{N}=4$ without any further computation. The only master integrals necessary for these configurations are pentagons and boxes, both of which have known closed forms in general dimension.

The basis we use was proposed by Giele, Kunszt, and Melnikov [65], and later used for the all-plus amplitude and other examples by Badger [42]; we re-derive it in section 3.1 for completeness. Techniques for fixing one-loop amplitudes by cuts in four-dimensions generalise to provide direct techniques to extract the coefficients of the scalar integrals forming the smaller basis up to $\mathcal{O}(\epsilon)$ and rational terms. In sections 3.2 and 3.3 we show how we can use an equivalent technique for the box and pentagon coefficients. Moreover, in the case of the box we see that the massive solutions can easily be parametrised in terms of the four-dimensional null solutions.

\subsection{Integral bases}

The well-known principle of reducing loop-level amplitudes to kinematic coefficients over a basis of known master-integrals takes a simple form at one-loop. When truncating to $\mathcal{O}(\epsilon)$, an amplitude $A_{n}$ can be expressed

$$
A_{n}=\mathbf{d}_{4} \cdot \mathbf{I}_{4}^{D=4-2 \epsilon}+\mathbf{d}_{\mathbf{3}} \cdot \mathbf{I}_{3}^{D=4-2 \epsilon}+\mathbf{d}_{\mathbf{2}} \cdot \mathbf{I}_{2}^{D=4-2 \epsilon}+d_{R}+\mathcal{O}(\epsilon) .
$$

This is a reduced version of a complete basis $[42,65,66]$ which includes pentagons, tadpoles and higher-dimensional scalar integrals

$$
\begin{aligned}
A_{n}=\mathbf{b}_{5} \cdot \mathbf{I}_{5}^{D=\bar{D}-2 \epsilon} & +\mathbf{b}_{4}(\epsilon) \cdot \mathbf{I}_{4}^{D=\{4,6,8\}-2 \epsilon}+\mathbf{b}_{\mathbf{3}}(\epsilon) \cdot \mathbf{I}_{3}^{D=\{4,6\}-2 \epsilon} \\
& +\mathbf{b}_{\mathbf{2}}(\epsilon) \cdot \mathbf{I}_{2}^{D=\{4,6\}-2 \epsilon}+\mathbf{b}_{\mathbf{1}}(\epsilon) \cdot \mathbf{I}_{1}^{D=4-2 \epsilon} .
\end{aligned}
$$


We leave $\bar{D}$ as a general integer placeholder to be fixed later in the derivation. The epsilon dependence of the coefficient in fact takes a very particular form, such that it integrates well with massive unitarity cuts. For completeness it is worth reviewing how this basis is derived [42].

The principles underlying the building of a basis are both to ensure that the basis is compatible with the generalised unitarity technique which builds the amplitude, in the sense that a given cut is precisely a prefactor of a given integral without overlaps or other ambiguities, and that it is general enough to capture all high-multiplicity behaviour. The most general basis one could write down includes all possible one-loop $4-2 \epsilon$ Feynman integrals with general numerator dependence on loop momentum $\ell$, as emerges naturally from a dimensionally regulated Feynman diagram construction in four dimensions:

$$
\left\{I_{m}^{4-2 \epsilon}[\mathcal{P}(\ell)]\right\} .
$$

There are further constraints we can apply to this polynomial. The theory we are working in is renormalisable. This puts a limit on the mass dimension of the vertices, and thus through power counting on the order of the polynomial $\mathcal{P}$ for a given one-loop graph. For example the bubble Feynman diagram depicted in figure 4 could only contribute numerators at worst quadratic in the loop momentum.

It is also very well known that basis elements possessing numerator terms involving the four-dimensional components of the loop momentum $l^{\mu}$ can be expressed in terms of scalar integrals $[43,78,79]$. On any given cut, this implies that the numerator $\mathcal{P}$ becomes a polynomial in the "mass" term $\mu^{2}$ from the $(-2 \epsilon)$-dimensional component of the momentum (see section 2). We choose to represent numerators in this form, which allows us to conclude that [49]

$$
I_{m}^{4-2 \epsilon}\left[\mu^{2 r}\right]=-\epsilon(1-\epsilon) \cdots(r-1-\epsilon) I_{m}^{4+2 r-2 \epsilon} .
$$

Although $r \leq\left\lfloor\frac{n}{2}\right\rfloor$ for diagrams of the type in figure 4, there is the question of how the power counting follows the reduction of $(m \geq 6)$-point integrals to pentagons. Moreover, using generalised unitarity to fix the coefficient of the pentagon integral functions leads to an ambiguity as to the dimension they are defined in.

This can be understood from the fact that the $\epsilon$-dependence of the coefficients in the integral dimension-shift identity [43]

$$
I_{5}^{D}=\frac{1}{2}\left[\sum_{j=1}^{n} c_{j} I_{4}^{D ;(j)}+(4-D) c_{0} I_{5}^{D+2}\right],
$$

is identical to that arising from a $\mu^{2}$ being inserted into the numerator of $I_{5}^{D}$. Applying the identity (3.5) into itself $r$ times gives

$$
\begin{aligned}
I_{5}^{4-2 \epsilon} & =-\epsilon(1-\epsilon) \cdots(r-1-\epsilon) I_{5}^{4+2 r-2 \epsilon}+\text { boxes } \\
& =I_{5}^{4-2 \epsilon}\left[\mu^{2 r}\right]+\text { boxes }
\end{aligned}
$$

so that the choice $r$ in this case is determined by the choice of dimension-shifted boxes that are included in the basis. Choosing to include boxes $I_{4}\left[\mu^{2 r}\right]$ implies that we can restrict 

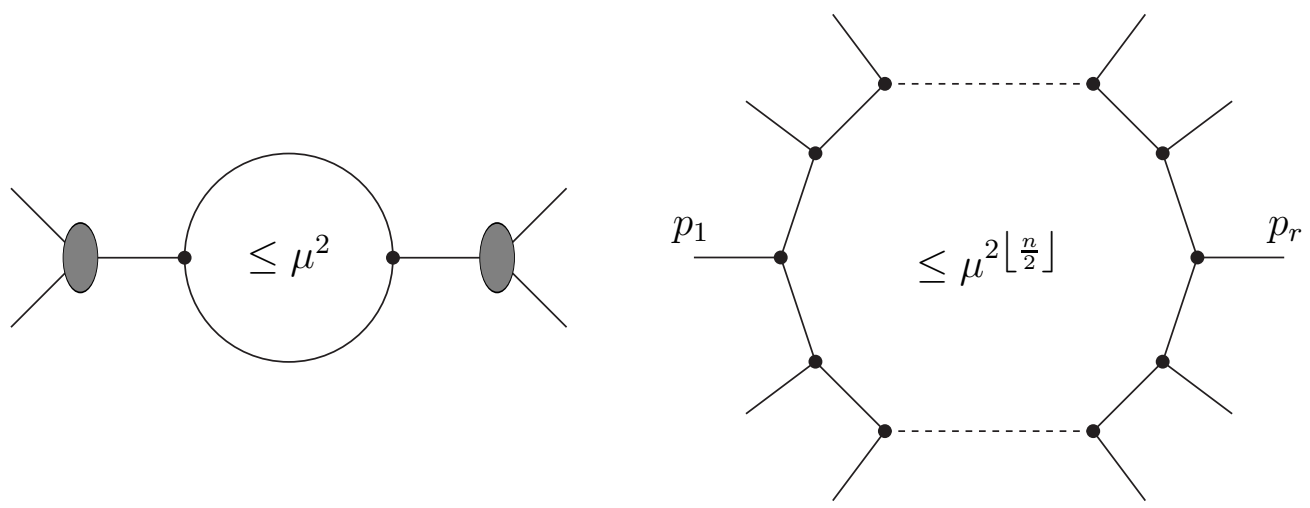

Figure 4. Ultraviolet limits on the bubble integral. Left is the possible sources of $\mu^{2 r}$ numerators in renormalisable gauge theories, each vertex contributing a possible $\ell^{\mu}$ to the numerator. The only possible contributions where $r>1$ comes from higher point integrals, which we include separately in the basis.

ourselves to pentagon coefficients of $I_{5}\left[\mu^{2 r+2}\right]$, as the box cuts compute the contribution from the first term on the right-hand side of equation (3.5) for that respective $r$. The choice of which boxes to include is thus contingent on the choice of pentagon, and the cut computes the independent contributions alongside the contribution from a pentagon shifted from 0 to $r+1$. As could be deduced from the box version of figure 4 , there is an upper limit of $r=2$ for a given box to exist independently from higher point functions. This implies the truncation of polynomials in $\mu^{2}$ to quadratic order $\left(\mu^{4}\right)$.

The external momenta are four-dimensional, and thus there is the additional simplification that $n$-point integrals reduce to pentagons for $n>5$. This fact can be seen in a number of ways [43], and recently a simple formula was written down by one of the authors [44] which generalises very simply for any $n$. Crucially, there is a very simple coincidence of interpretation with maximal unitarity cuts, which allows the coefficient of the reduction to simply be interpreted as propagators frozen at a cut solution. This compatibility permits us to interpret, for $m>5$,

$$
\left.I_{m}^{4-2 \epsilon}\right|_{\left[i_{1}, i_{2}, i_{3}, i_{4}, i_{5}\right] \text { cut }}=\left[\prod_{j=1}^{m-5} \xi_{j}\right] I_{5}^{\left[i_{1}, i_{2}, i_{3}, i_{4}, i_{4}\right] ; 4-2 \epsilon},
$$

so capturing the pentagons will also reproduce all higher-point integrals, thus extending generalised unitarity to treat complete unitarity cuts. We emphasise here that all degrees of freedom are fixed, so unlike the box case $\mu^{2}$ is not a degree of freedom for the $(m \geq 5)$-point integrals. The general solution to the penta-cut is [44]

$$
\begin{aligned}
\left.\mu^{2}\right|_{\left[i_{1}, i_{2}, i_{3}, i_{4}, i_{5}\right] \text { cut }} & =\frac{1}{c_{0}}=\frac{16 \Upsilon_{5}}{\Delta_{5}} \\
& =\frac{\operatorname{tr}\left(q_{i_{1} i_{2}} q_{i_{2} i_{3}} q_{i_{3} i_{4}} q_{i_{4} i_{5}} q_{i_{5} i_{1}} q_{i_{1} i_{2}} q_{i_{2} i_{3}} q_{i_{3} i_{4}} q_{i_{4} i_{5}} q_{i_{5} i_{1}}\right)-2 \prod_{k=1}^{5} q_{i_{k} i_{k+1}}^{2}}{\operatorname{tr}_{5}^{2}\left(q_{i_{1} i_{2}} q_{i_{2} i_{3}} q_{i_{3} i_{4}} q_{i_{4} i_{5}}\right)} .
\end{aligned}
$$

Thus we can refine the basis (3.2), as we know what the $\epsilon$-dependence of the coefficients must be, and so we bundle the dependence into the integral basis. Knowing that boxes can 
arise independently for $I_{m}\left[\mu^{2 r}\right]$, we also fix the dimension of the pentagon to $D=10-2 \epsilon$ (i.e. $r=3$ ) such that boxes are included up to $I_{4}\left[\mu^{4}\right]$ separately in the basis. For massless theories we drop tadpoles; our final basis is thus

$$
A_{n}=\mathbf{b}_{5} \cdot \mathbf{I}_{5}\left[\mu^{6}\right]+\mathbf{b}_{4} \cdot \mathbf{I}_{4}\left[1, \mu^{2}, \mu^{4}\right]+\mathbf{b}_{3} \cdot \mathbf{I}_{3}\left[1, \mu^{2}\right]+\mathbf{b}_{2} \cdot \mathbf{I}_{2}\left[1, \mu^{2}\right] .
$$

The ingredients needed to construct the coefficients are the tree amplitudes in equation (2.14) which we use to compute the one-loop amplitude $A_{n}^{\mathrm{AP}}=2 A_{n}^{[0]}$. The bubbles and triangles vanish trivially from the scaling of the tree amplitudes

$$
A^{\text {tree }}\left(\mathbf{1}^{0}, 2^{+}, 3^{+}, \ldots,(n-1)^{+}, \mathbf{n}^{0}\right) \sim \mu^{2}
$$

so that a nonvanishing product of tree amplitudes would contribute only to order $\mathcal{O}\left(\mu^{4}\right)$, which is entirely captured by the box integrals, by the above arguments. In other words, we can set $\mathbf{b}_{3}=\mathbf{b}_{2}=0$ in equation (3.9). We will proceed to show that the coefficients of $\mathbf{I}_{4}\left[\mu^{4}\right]$ vanish as well.

\subsection{The cut box}

To compute the box coefficients which compose $\mathbf{b}_{4}^{\mathrm{AP}}$ we use a method analogous to how generalised four-dimensional cuts can be used to determine the coefficient of the $\mathcal{N}=4$ MHV amplitude.

The four-dimensional cut conditions for massless propagators

$$
\ell_{i_{k}}^{2}=l_{i_{k}}^{2}=0, \quad k \in\{1,2,3,4\}
$$

are readily solved. For the massless, one-mass and two-mass boxes depicted in the top row in figure 5 the solution to the conditions (3.11) is

$$
l_{i_{1}}^{\mu}=\bar{l}_{ \pm}^{\mu}=\frac{\operatorname{tr}_{ \pm}\left(q_{i_{1} i_{2}} i_{2} i_{4} \gamma^{\mu}\right)}{s_{i_{2} i_{4}}}
$$

which can also be expressed in spinor-helicity formalism as

$$
\begin{aligned}
& \left(\bar{l}_{+}\right)_{a \dot{b}}=\left(\frac{\left.\mid i_{4}\right]\left[i_{2} \mid q_{i_{1} i_{2}}\right.}{\left[i_{2} i_{4}\right]}\right)_{a \dot{b}}, \\
& \left(\bar{l}_{-}\right)_{a \dot{b}}=\left(\frac{q_{i_{1} i_{2}}\left|i_{2}\right\rangle\left\langle i_{4}\right|}{\left\langle i_{4} i_{2}\right\rangle}\right)_{a \dot{b}} .
\end{aligned}
$$

For massive propagators, the cut conditions are

$$
\ell_{i_{k}}^{2}=l_{i_{k}}^{2}-\mu^{2}=0, \quad k \in\{1,2,3,4\},
$$

which have an extra unfixed parameter compared to the massless cut conditions in equation (3.11). Intuitively, to match up with the basis (3.9), this parameter can simply be $\mu^{2}$, however a more generally applicable solution is to choose it to be a dimensionless parameter $\alpha$, such that we can define the solution to equations (3.14) in terms of the massless solutions (3.12):

$$
l_{1}^{\mu}=\left(\alpha \bar{l}_{+}+(1-\alpha) \bar{l}_{-}\right)^{\mu}
$$


with the relationship between $\alpha$ and $\mu$ defined by

$$
\alpha(1-\alpha)=\frac{\mu^{2}}{2 l_{+} \cdot l_{-}} .
$$

It is convenient to note that $2 l_{+} \cdot l_{-}=\left(c_{0}^{\text {box }}\right)^{-1}$ for the following representation ${ }^{10}$ of $c_{0}^{\text {box }}$,

$$
c_{0}^{\text {box }}=\frac{4 s_{i_{2} i_{4}}}{\left[i_{2}\left|q_{i_{1} i_{2}}\right| i_{2}\right\rangle\left[i_{4}\left|q_{i_{1} i_{2}}\right| i_{4}\right\rangle} .
$$

We can then write solutions to equation (3.16) as

$$
\alpha_{ \pm}=\frac{1 \pm \sqrt{1+c_{0}^{\mathrm{box}} \mu^{2}}}{2} .
$$

The solution to (3.14) is split into two branches which meet at $\alpha=\frac{1}{2}$. The two solutions simply correspond to the choice of parametrisation, as it is trivial to see that the replacement

$$
\alpha \rightarrow 1-\alpha, \quad \pm \rightarrow \mp
$$

leaves (3.15) unaltered. When making the choice, one needs to average over the solutions, just like for the massless case. Instead of making the solution depend on choice of $\alpha_{ \pm}$we fix $\alpha \equiv \alpha_{+}$then our two solutions can be expressed

$$
l_{ \pm}=\alpha \bar{l}_{ \pm}+(1-\alpha) \bar{l}_{\mp} .
$$

These solutions are then input into the massive cuts of the kind depicted in figure 5 .

As the three-point amplitude with one on-shell gluon and two equal-mass scalars is not defined by equation (2.14), we define it here [51, 77]:

$$
A_{3}^{\text {tree }}\left(i^{+}, l_{i+1}^{0}, l_{i}^{0}\right)=\frac{\left[i\left|l_{i}\right| \chi\right\rangle}{\langle i \chi\rangle} .
$$

We see that unlike the leading term of the four-point and above UHV amplitudes, the amplitude (3.21) does not scale linearly with $\mu^{2}$. As with the bubbles and triangles, we will use the truncation of the $\mu^{2}$ polynomial to rule out two-mass hard, three-mass and four-mass boxes which form the bottom diagrams of figure 5 from the functional form. To this end, and to simplify future manipulations, we explicitly consider some basic simplifications of adjacent three-point amplitudes. Without loss of generality considering the adjacent massive cuts $l_{1}^{2}=l_{2}^{2}=l_{3}^{2}=\mu^{2}$; we can express the corresponding product of amplitudes thus

$$
\begin{aligned}
A_{3}^{\text {tree }}\left(1^{+},-l_{1}^{0}, l_{2}^{0}\right) \times A_{3}^{\text {tree }}\left(2^{+},-\boldsymbol{l}_{2}^{0}, \boldsymbol{l}_{4}^{0}\right) & =-\frac{\left[1\left|l_{1}\right| \chi\right\rangle}{\langle 1 \chi\rangle} \times \frac{\left[2\left|l_{1}\right| \chi\right\rangle}{\langle 2 \chi\rangle} \\
& =\frac{\left\langle\chi\left|l_{1} 12 l_{1}\right| \chi\right\rangle}{\langle 1 \chi\rangle\langle 2 \chi\rangle\langle 12\rangle} \\
& =\mu^{2} \frac{[12]}{\langle 12\rangle}
\end{aligned}
$$

\footnotetext{
${ }^{10}$ The box coefficient can also be expressed as a ratio of kinematic determinants $[43,44,80]$.
} 

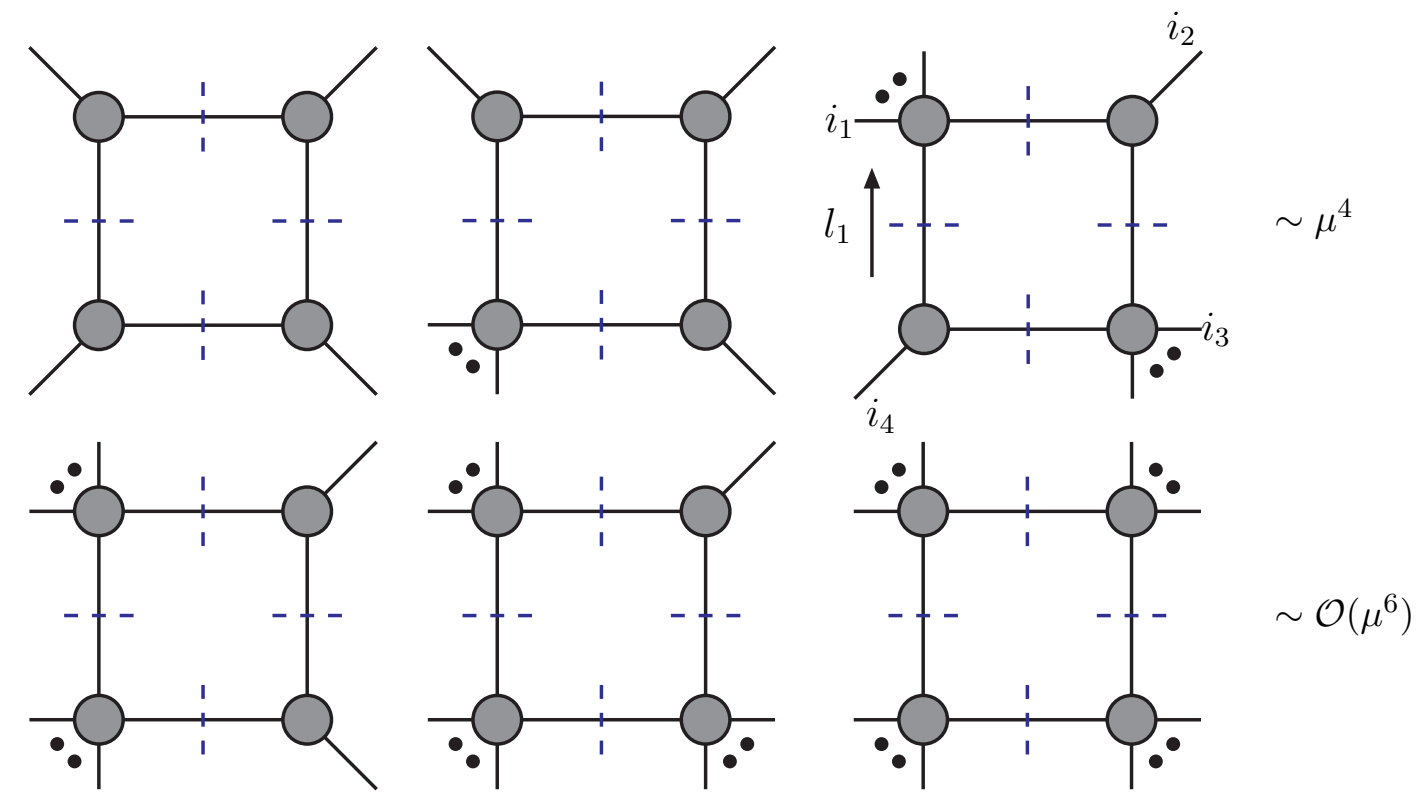

Figure 5. Top: diagrams which contribute to the basis (3.9). Bottom: diagrams which are eliminated by the truncation of terms $\mathcal{O}\left(\mu^{6}\right)$.

where we have made use of the fact that the cut conditions imply $l_{1} \cdot p_{1}=l_{1} \cdot p_{2}=0$ to commute the $l_{1}$ s together in the last line using the identity

$$
\langle X|\{a, b\}| Y\rangle=2 p_{a} \cdot p_{b}\langle X Y\rangle .
$$

Equation (3.22) implies that, like $n \geq 4$-point off-shell amplitudes, adjacent threepoint vertices have an $\mathcal{O}\left(\mu^{2}\right)$ scaling, implying that two-mass hard boxes have polynomials which start at $\mathcal{O}\left(\mu^{6}\right)$, like three-mass boxes; four-mass boxes begin at $\mathcal{O}\left(\mu^{8}\right)$. Recalling that our choice of basis in equation (3.9) implies the truncation of this polynomial up to $\mathcal{O}\left(\mu^{4}\right)$, we assert that, much like the massless $\mathcal{N}=4$ cuts, the all-plus scalar cuts only have contributions from two-mass-easy, one-mass and, in the four-point case, massless boxes.

The coefficients of the boxes are captured by inputting the cut solutions given in equation (3.15) into the appropriate amplitudes, averaging over $\alpha_{ \pm}$and truncating terms $\mathcal{O}\left(\mu^{6}\right)$. Because of this truncation, the computation is greatly simplified, as generically from the definition of amplitudes with two internal scalars (2.14),

$A^{\text {tree }}\left(-\boldsymbol{l}_{i_{1}}^{0}, i_{1}^{+}, \ldots,\left(i_{2}-1\right)^{+}, \boldsymbol{l}_{i_{2}}^{0}\right)=\frac{\mu^{2}\left[i_{1}\left|l_{i_{1}} q_{i_{1} i_{2}}\right| i_{2}-1\right]+\mathcal{O}\left(\mu^{4}\right)}{\left(s_{l_{i_{1}} i_{1}}-\mu^{2}\right)\left\langle i_{1}\left(i_{1}+1\right) \cdots\left(i_{2}-2\right)\left(i_{2}-1\right)\right\rangle\left(s_{l_{i_{2}}\left(i_{2}-1\right)}-\mu^{2}\right)}$,

so we can truncate terms $\sim \mathcal{O}\left(\mu^{4}\right)$, as they contribute to the cut to $\mathcal{O}\left(\mu^{6}\right)$ at most. We double our expression of the coefficient $b_{4}^{\left[i_{1}, i_{3}-1, i_{3}, i_{1}-1\right]}$ of the $8-2 \epsilon$ dimensional two-masseasy box integral $I_{4}^{\left[i_{1}, i_{3}-1, i_{3}, i_{1}-1\right]}\left[\mu^{4}\right]$ as $A_{n}^{A P}=2 A^{[0]}$ with the all-plus configuration

$$
\begin{aligned}
b_{4}^{\left[i_{1}, i_{3}-1, i_{3}, i_{1}-1\right]} & =\frac{1}{\mu^{4}}\left[2 \times \frac{1}{2} \sum_{\alpha_{ \pm}} A^{\text {tree }} \times A^{\text {tree }} \times A^{\text {tree }} \times\left. A^{\text {tree }}\right|_{\mathcal{O}\left(\mu^{6}\right)}\right] \\
& =\frac{1}{2} \frac{\operatorname{tr}\left(i_{1} q_{i_{1} i_{3}} i_{3} q_{i_{3}+1, i_{1}-1}\right)}{\langle 12 \ldots n 1\rangle},
\end{aligned}
$$




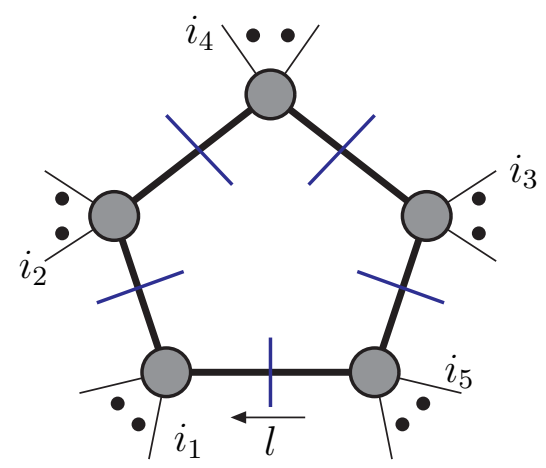

Figure 6. The pentagon cut.

where $\left.\right|_{\mathcal{O}\left(\mu^{6}\right)}$ denotes truncation of terms $\mathcal{O}\left(\mu^{6}\right)$; we suppress the arguments of the two-scalar all-plus tree amplitudes in the cut. The result (3.25) matches the expected coefficient from the shifted version of the $\mathcal{N}=4$ coefficient of the box given in equation (1.26).

\subsection{Pentagon coefficients}

We introduce the maximal cut unitarity solution to solve for the pentagon coefficient. The solution is remarkably compact for all descendant pentagons [44]

The five-particle maximal cut depicted in figure 6 fixes the loop momentum completely:

$$
\begin{gathered}
l_{i_{1}}^{\mu}=-\frac{\operatorname{tr}_{5}\left(q_{i_{1} i_{2}} q_{i_{2} i_{3}} q_{i_{3} i_{4}} q_{i_{4} i_{5}} q_{i_{5} i_{1}} \gamma_{\mu}\right)}{2 \operatorname{tr}_{5}\left(q_{i_{1} i_{2}} q_{i_{2} i_{3}} q_{i_{3} i_{4}} q_{i_{4} i_{5}}\right)} \\
\mu^{2}=c_{0}^{-1}=\frac{\operatorname{tr}\left(q_{i_{1} i_{2}} q_{i_{2} i_{3}} q_{i_{3} i_{4}} q_{i_{4} i_{5}} q_{i_{5} i_{1}} q_{i_{1} i_{2}} q_{i_{2} i_{3}} q_{i_{3} i_{4}} q_{i_{4} i_{5}} q_{i_{5} i_{1}}\right)-2 \prod_{k=1}^{5} q_{i_{k} i_{k+1}}^{2}}{\operatorname{tr}_{5}^{2}\left(q_{i_{1} i_{2}} q_{i_{2} i_{3}} q_{i_{3} i_{4}} q_{i_{4} i_{5}}\right)} .
\end{gathered}
$$

One now need simply draw all possible pentagons. The coefficient computed from the product of amplitudes is naturally interpreted as that of the four-dimensional pentagon $I_{5}[1]$, but as we have already computed box coefficients up to $I_{4}\left[\mu^{4}\right]$, part of this function's contribution to the amplitude has already been captured. Applying the dimension-shift relation (3.5) three times up to $I_{5}\left[\mu^{6}\right]$ introduces a factor $c_{0}^{3}$. The coefficient of $I_{5}^{\left[i_{1}, i_{2}, i_{3}, i_{4}, i_{5}\right]}\left[\mu^{6}\right]$ is thus

$$
\begin{array}{r}
b_{5}^{\left[i_{1}, i_{2}, i_{3}, i_{4}, i_{5}\right]}=c_{0}^{3} A^{\text {tree }}\left(-\boldsymbol{l}_{i_{1}}^{0}, i_{1}^{+}, \ldots,\left(i_{2}-1\right)^{+}, \boldsymbol{l}_{i_{2}}^{0}\right) \times A^{\text {tree }}\left(-\boldsymbol{l}_{i_{2}}^{0}, i_{2}^{+}, \ldots,\left(i_{3}-1\right)^{+}, \boldsymbol{l}_{i_{3}}^{0}\right) \times \\
A^{\text {tree }}\left(-\boldsymbol{l}_{i_{3}}^{0}, i_{3}^{+}, \ldots,\left(i_{4}-1\right)^{+}, \boldsymbol{l}_{i_{3}}^{0}\right) \times A^{\text {tree }}\left(-\boldsymbol{l}_{i_{4}}^{0}, i_{4}^{+}, \ldots,\left(i_{5}-1\right)^{+}, \boldsymbol{l}_{i_{3}}^{0}\right) \times \\
A^{\text {tree }}\left(-\boldsymbol{l}_{i_{5}}^{0}, i_{5}^{+}, \ldots,\left(i_{1}-1\right)^{+}, \boldsymbol{l}_{i_{3}}^{0}\right) .
\end{array}
$$

Note that propagator factors can be substituted for the general $(n>5)$-multiplicity kinematic coefficients of the integral reduction $I_{n} \rightarrow I_{5}[44]$

$$
\left.\frac{1}{q_{-l_{i_{k}} r}^{2}-\mu^{2}}\right|_{\left[i_{1}, i_{2}, i_{3}, i_{4}, i_{5}\right] \mathrm{cut}}=\frac{1}{2} \xi_{r}^{\left[i_{1}, i_{2}, i_{3}, i_{4}, i_{5}, r\right]},
$$


where

$$
\xi_{r}^{\left[i_{1}, i_{2}, i_{3}, i_{4}, i_{5}, r\right]}=\frac{-2 \operatorname{tr}_{5}\left(q_{i_{1} i_{2}} q_{i_{2} i_{3}} q_{i_{3} i_{4}} q_{i_{4} i_{5}}\right)}{\operatorname{tr}_{5}\left(q_{i_{1} i_{2}} q_{i_{2} i_{3}} q_{i_{3} i_{4}} q_{i_{4} i_{5}} q_{i_{5} r} q_{r i_{1}}\right)} .
$$

With the solution (3.26), the substitution (3.28) and the explicit form of the off-shell scalar amplitude (2.14), equation (3.27) is a closed form for any given pentagon coefficient.

\subsection{Six-point example}

We demonstrate the technique explicitly by computing the first non-trivial example: the six-point amplitude $A_{6}^{\mathrm{AP}}$. There are two possible box configurations, one mass and twomass-easy. The box cuts are thus of the form

$$
\begin{aligned}
& b_{4}^{[a, b, c, f]}=\left.\frac{1}{\mu^{4}} \sum_{l_{ \pm}}\left[A_{3}^{\text {tree }} \times A_{3}^{\text {tree }} \times A_{5}^{\text {tree }} \times A_{3}^{\text {tree }}\right]\right|_{\mathcal{O}\left(\mu^{6}\right)}, \\
& b_{4}^{[a, c, d, f]}=\left.\frac{1}{\mu^{4}} \sum_{l_{ \pm}}\left[A_{4}^{\text {tree }} \times A_{3}^{\text {tree }} \times A_{4}^{\text {tree }} \times A_{3}^{\text {tree }}\right]\right|_{\mathcal{O}\left(\mu^{6}\right)} .
\end{aligned}
$$

We can simplify the computation by massaging these expressions before inputting the explicit solutions $l_{ \pm}$defined in equation (3.15). Using the identity (3.22)

$$
\mu^{4} b_{4}^{[a, b, c, f]}=\left.\sum_{l_{ \pm}}\left[\mu^{2} \frac{[f a]}{\langle f a\rangle} \times \frac{\mu^{2}\left[c\left|-l_{c} q_{c e}\right| e\right]\left[b\left|l_{c}\right| \chi\right\rangle}{\left(s_{-l_{c} c}-\mu^{2}\right)\langle c d\rangle\langle d e\rangle\langle b \chi\rangle\left(s_{e l_{f}}-\mu^{2}\right)}\right]\right|_{\mathcal{O}\left(\mu^{6}\right)},
$$

where the left-most factor is a combination of the two three-point amplitudes with legs $f$ and $a$. If we factor out the Parke-Taylor denominator, we can concatenate the numerator

$$
\mu^{4} b_{4}^{[a, b, c, f]}=\left.\frac{1}{\langle 12 \ldots 61\rangle} \sum_{l_{ \pm}}\left[\frac{\mu^{4}\left\langle\chi\left|l_{c} b c\left(-l_{c}\right) q_{c e} e f a\right| b\right\rangle}{\left(s_{-l_{c} c}-\mu^{2}\right)\langle b \chi\rangle\left(s_{e l_{f}}-\mu^{2}\right)}\right]\right|_{\mathcal{O}\left(\mu^{6}\right)} .
$$

By noting that

$$
\begin{aligned}
s_{-l_{c} c}-\mu^{2} & =-2 l_{c} \cdot p_{c}, \\
2 l_{c} \cdot p_{b} & =l_{b}^{2}-\mu^{2}=0,
\end{aligned}
$$

we can commute the $l_{c}$ through up to $l_{c} \cdot l_{c}=\mu^{2}$

$$
\mu^{4} b_{4}^{[a, b, c, f]}=\left.\frac{1}{\langle 12 \ldots 61\rangle} \sum_{l_{ \pm}}\left[\frac{\mu^{4}\left[b\left|\left(-l_{c}\right) q_{c e} e f a\right| b\right\rangle}{\left(s_{e l_{f}}-\mu^{2}\right)}+\mathcal{O}\left(\mu^{6}\right)\right]\right|_{\mathcal{O}\left(\mu^{6}\right)}
$$

and upon noting that by commuting $l_{c}$ through all the other terms in $[b|\cdots| b\rangle=\operatorname{tr}_{+}(b \cdots)$

$$
\begin{aligned}
{\left[b\left|\left(-l_{c}\right) q_{c e} e f a\right| b\right\rangle } & =-2 l_{c} \cdot q_{c e}[b|a f a| b\rangle-\left[b\left|\left(-l_{c}\right) q_{c e} e f a\right| b\right\rangle \\
& =\frac{1}{2}\left(s_{e l_{f}}-\mu^{2}\right) s_{f a} s_{a b},
\end{aligned}
$$


the one-mass box coefficient falls out without explicitly entering the solutions $l_{ \pm}$, as

$$
b_{4}^{[a, b, c, f]}=-\frac{1}{\langle 12 \ldots 61\rangle} s_{f a} s_{a b}=\frac{1}{2} \operatorname{tr}\left(f a b q_{c f}\right) .
$$

The two-mass box coefficients can be simplified in a similar way, but to diversify the demonstration we show how to manipulate the expression with the $\alpha$ form of solution from equation (3.15).

$$
\begin{aligned}
\mu^{4} b_{4}^{[a, c, d, f]}= & \left.\sum_{l_{ \pm}}\left[\frac{\mu^{2}[a b]}{\langle a b\rangle\left(s_{-l_{a} a}-\mu^{2}\right)} \times \frac{\left\langle\chi\left|l_{c}\right| c\right]}{\langle c \chi\rangle} \times \frac{\mu^{2}[d e]}{\langle d e\rangle\left(s_{-l_{d} d}-\mu^{2}\right)} \times \frac{\left\langle\chi\left|l_{f}\right| f\right]}{\langle f \chi\rangle}\right]\right|_{\mathcal{O}\left(\mu^{6}\right)} \\
= & \frac{\mu^{4}[a b][d e]}{\langle a b\rangle\langle d e\rangle}\left[\frac{\alpha\left[c\left|f q_{a c}\right| c\right] \alpha\left[f\left|c q_{d f}\right| f\right]}{4 s_{a f}^{2}\left(2\left(\alpha \bar{l}_{d+}+(1-\alpha) \bar{l}_{d-}\right) \cdot p_{d}\right)\left(2\left(\alpha \bar{l}_{a+}+(1-\alpha) \bar{l}_{a-}\right) \cdot p_{a}\right)}+\right. \\
& \left.\frac{(1-\alpha)\left[c\left|f q_{a c}\right| c\right](1-\alpha)\left[f\left|c q_{d f}\right| f\right]}{4 s_{a f}^{2}\left(2\left((1-\alpha) \bar{l}_{d+}+\alpha \bar{l}_{d-}\right) \cdot p_{d}\right)\left(2\left((1-\alpha) \bar{l}_{a+}+\alpha \bar{l}_{a-}\right) \cdot p_{a}\right)}\right]\left.\right|_{\mathcal{O}\left(\mu^{6}\right)} ;
\end{aligned}
$$

we can simply take the $\mu^{2} \rightarrow 0$ limit for the content of the bracket which implies, if we choose $\alpha=\alpha_{+}$, that $\alpha \rightarrow 1$

$$
\begin{aligned}
\mu^{4} b_{4}^{[a, c, d, f]} & =\left.\frac{\mu^{4}[a b][d e]}{\langle a b\rangle\langle d e\rangle}\left[\frac{\left[c\left|f q_{a c}\right| c\right]\left[f\left|c q_{d f}\right| f\right]}{4 s_{a f}^{2}\left(2 \bar{l}_{d+} \cdot p_{d} 2 \bar{l}_{a+} \cdot p_{a}\right)}+\mathcal{O}\left(\mu^{2}\right)\right]\right|_{\mathcal{O}\left(\mu^{6}\right)} \\
& =\left.\frac{\mu^{4}[a b][d e]}{\langle a b\rangle\langle d e\rangle}\left[\frac{\left[c\left|f q_{a c}\right| c\right]\left[f\left|c q_{d f}\right| f\right]}{\left\langle f\left|q_{d f} d c\right| f\right]\left\langle c\left|q_{a c} a f\right| c\right]}+\mathcal{O}\left(\mu^{2}\right)\right]\right|_{\mathcal{O}\left(\mu^{6}\right)} \\
& =\left.\frac{\mu^{4}}{\langle 12 \ldots 61\rangle}\left[\left\langle f\left|q_{a c}\right| c\right]\left\langle c\left|q_{d f}\right| f\right]+\mathcal{O}\left(\mu^{2}\right)\right]\right|_{\mathcal{O}\left(\mu^{6}\right)}
\end{aligned}
$$

thus, the coefficient is

$$
b_{4}^{[a, c, d, f]}=\frac{1}{2} \frac{\operatorname{tr}\left(c q_{a c} f q_{d f}\right)}{\langle 12 \ldots 61\rangle} .
$$

The penta-cut in equation (3.27) can be used to compute the pentagon coefficient

$$
b_{5}^{[a, b, c, d, e]}=c_{0}^{3} \frac{\left[a\left|l_{a}\right| \chi\right\rangle}{\langle a \chi\rangle} \times \frac{\left[b\left|l_{b}\right| \chi\right\rangle}{\langle b \chi\rangle} \times \frac{\left[c\left|l_{c}\right| \chi\right\rangle}{\langle c \chi\rangle} \times \frac{\left[d\left|l_{d}\right| \chi\right\rangle}{\langle d \chi\rangle} \times \frac{\mu^{2}[e f]}{\langle e f\rangle\left(s_{-l_{e} e}-\mu^{2}\right)} ;
$$

using the adjacent-three-point identity (3.22) substituting in the solution (3.26) gives

$$
\begin{aligned}
b_{5}^{[a, b, c, d, e]} & =\frac{[a b]}{\langle a b\rangle} \times \frac{[c d]}{\langle c d\rangle} \times \frac{[e f]}{\langle e f\rangle} \times \frac{1}{2} \xi_{f} \\
& =\frac{1}{\langle 12 \ldots 61\rangle} \operatorname{tr}_{+}(a b c d e f) \frac{1}{2} \xi_{f} \\
& =\frac{1}{\langle 12 \ldots 61\rangle}\left(\operatorname{tr}(123456) \frac{1}{2} \xi_{f}-\operatorname{tr}_{5}(a b c d)\right)
\end{aligned}
$$


where upon summing over cycles the hexagon function can be reconstructed from the first term in the final line.

Putting all the coefficients together reconstructs the full amplitude

$$
\begin{aligned}
A_{6}^{\mathrm{AP}}= & \frac{1}{\langle 12 \ldots 61\rangle}\left[-\sum_{1<j_{1}<j_{2} \leq n}^{n} \operatorname{tr}\left(j_{1} q_{j_{1}+1, j_{2}-1} j_{2} q_{j_{2}+1, j_{1}-1}\right) I_{4}^{\left(j_{1}, j_{2}\right)}\left[\mu^{4}\right]\right. \\
& \left.+\sum_{j=1}^{n} \operatorname{tr}_{5}(j+1, j+2, j+3, j+4) I_{5}^{(j)}\left[\mu^{6}\right]+\operatorname{tr}(123456) I_{6}\left[\mu^{6}\right]\right],
\end{aligned}
$$

which matches equation (1.12) upon shifting of the integrals $I\left[\mu^{2 r}\right]$ using equation (3.4).

\subsection{Reduction to finite limit}

To verify our results we can check that taking the limit $\epsilon \rightarrow 0$ reproduces known results. This is a strong check, as delicate cancellations need to occur between integral coefficients which do not follow obviously from their functional form.

The AP amplitude, to all orders in epsilon, is

$$
A_{n}^{\mathrm{AP}}=\sum_{\mathcal{P}_{4}^{2 m e} \subset\{1, \ldots, n\}} b_{4}^{\mathcal{P}} I_{4}^{\mathcal{P}}\left[\mu^{4}\right]+\sum_{\mathcal{P}_{5} \subset\{1, \ldots, n\}} b_{5}^{\mathcal{P}} I_{5}^{\mathcal{P}}\left[\mu^{6}\right],
$$

where $\mathcal{P}_{4}^{2 m e}$ consists of the "two-mass-easy" ordered subsets of $\{1, \ldots, n\}$ of the form

$$
\left\{i_{1}, i_{3}-1, i_{3}, i_{1}-1\right\}
$$

the subsets $\mathcal{P}_{5}$ are all five-element subsets of $\{1, \ldots, n\}$, and the $b_{4}^{\mathcal{P}}$ and $b_{5}^{\mathcal{P}}$ are defined in equations (3.25) and (3.27) respectively. The $\epsilon \rightarrow 0$ limit should yield

$$
A_{n}^{\mathrm{AP}}=\sum_{1 \leq i_{1}<i_{2}<i_{3}<i_{4} \leq n} \frac{\operatorname{tr}_{-}\left(i_{1} i_{2} i_{3} i_{4}\right)}{\langle 12 \ldots n 1\rangle}+\mathcal{O}(\epsilon) .
$$

Upon making the decomposition $\operatorname{tr}_{-}=\frac{1}{2}\left(\operatorname{tr}-\operatorname{tr}_{5}\right)$ and taking into account the limits of the integral

$$
\begin{aligned}
& \epsilon(1-\epsilon) I_{4}=\frac{1}{6}+\mathcal{O}(\epsilon), \\
& \epsilon(1-\epsilon) I_{5}=\frac{1}{24}+\mathcal{O}(\epsilon),
\end{aligned}
$$

then as shown by BDDK [23]

$$
\sum_{\mathcal{P}_{4}^{2 m e} \subset\{1, \ldots, n\}} b_{4}^{\mathcal{P}} I_{4}^{\mathcal{P}}\left[\mu^{4}\right]=\sum_{1 \leq i_{1}<i_{2}<i_{3}<i_{4} \leq n} \frac{\operatorname{tr}\left(i_{1} i_{2} i_{3} i_{4}\right)}{2\langle 12 \ldots n 1\rangle} .
$$

Equally it must hold true that

$$
\sum_{\mathcal{P}_{5} \subset\{1, \ldots, n\}} b_{5}^{\mathcal{P}} I_{5}^{\mathcal{P}}\left[\mu^{6}\right]=-\sum_{1 \leq i_{1}<i_{2}<i_{3}<i_{4} \leq n} \frac{\operatorname{tr}_{5}\left(i_{1} i_{2} i_{3} i_{4}\right)}{2\langle 12 \ldots n 1\rangle} .
$$

This does not appear to fall out naturally from the functional forms of the coefficients $b_{5}^{\mathcal{P}}$, but as the solutions and functional form are completely determined, it provides a nontrivial check on the validity of the expression (3.27). We confirm that this holds numerically up to $n=17$. 


\subsection{Transcendental structure}

For $n=4,5$, and before performing the dimension shift, the MHV amplitude is a pure function in the sense of [81]. That is to say, taking the $n=5$ case in particular, it can be written in the form

$$
A_{5}^{\mathcal{N}=4}\left(1^{-}, 2^{-}, 3^{+}, 4^{+}, 5^{+}\right)=A_{5}^{\text {tree }}\left(1^{-}, 2^{-}, 3^{+}, 4^{+}, 5^{+}\right) f_{5}^{\mathrm{MPL}}\left(\left\{s_{i, i+1}\right\}, \epsilon\right),
$$

where $f_{5}^{\mathrm{MPL}}$ is a linear combination of multiple polylogarithms without any additional kinematic coefficients. This can be seen by noticing that the coefficients appearing inside the brackets in equation (1.11) precisely cancel the leading singularities of the corresponding integrals, leaving the normalized versions of $I_{4}^{4-2 \epsilon}, I_{5}^{6-2 \epsilon}$, which belong to the uniformly transcendental basis used in [82].

To see whether the pattern holds at $n=6$, we must check the leading singularity of the hexagon integral in equation (1.12). The amplitude before the dimension shift is

$$
\begin{aligned}
A_{6}^{\mathcal{N}=4}= & \frac{\langle i j\rangle^{4}}{4\langle 12 \ldots 61\rangle}\left[-\sum_{j_{1}, j_{2}=1}^{n} \operatorname{tr}\left(j_{1} q_{j_{1}+1, j_{2}-1} j_{2} q_{j_{2}+1, j_{1}-1}\right) I_{4}^{4-2 \epsilon ;\left(j_{1}, j_{2}\right)}\right. \\
& \left.-2 \epsilon\left(\sum_{j=1}^{n} \operatorname{tr}_{5}(j+1, j+2, j+3, j+4) I_{5}^{6-2 \epsilon,(j)}+\operatorname{tr}(123456) I_{6}^{6-2 \epsilon}\right)\right] .
\end{aligned}
$$

Similarly to the five-point case, the boxes and pentagon integrals are multiplied by the coefficients cancelling their leading singularities, $\operatorname{tr}\left(j_{1} q_{j_{1}+1, j_{2}-1} j_{2} q_{j_{2}+1, j_{1}-1}\right)$ and $\operatorname{tr}_{5}(j+$ $1, j+2, j+3, j+4)$ respectively. ${ }^{11}$ The hexagon breaks down entirely to pentagons [43, 44]

$$
I_{6}=\frac{1}{2} \sum_{j=1}^{6} \xi_{j} I_{5}^{(j)}
$$

where

$$
\xi_{j}=2 \frac{(-1)^{j} \operatorname{tr}_{5}(j+1, j+2, j+3, j+4)}{\operatorname{tr}_{5}(123456)}
$$

and thus the purity is broken by terms with the rational prefactor

$$
(-1)^{j} \frac{\operatorname{tr}(123456)}{\operatorname{tr}_{5}(123456)}
$$

where the denominator is the leading singularity ${ }^{12}$ of the hexagon. It should also be highlighted that the dimension shifted hexagon does not contribute to the finite rational $\left(\epsilon^{0}\right)$ piece of the AP amplitude as

$$
\epsilon(1-\epsilon) I_{6}^{8-2 \epsilon}=\mathcal{O}(\epsilon) .
$$

\footnotetext{
${ }^{11}$ See [44] for representations of these reduction coefficients as determinants.

${ }^{12}$ As the hexagon breaks down entirely to pentagons, the notion of it having a leading singularity could be considered dubious. Here we use the same definition as in [82], with a natural representation as a kinematic determinant.
} 
Thus while the MHV amplitude fails to preserve purity at $n=6$, it is still possible to match the pure structure to the finite contribution of the AP amplitude. However, this matching becomes more complicated for $n \geq 7$.

The fact that MHV integrands in $\mathcal{N}=4 \mathrm{SYM}$ have dlog forms $[4,83]$ led to the hope that the integrated expressions would be pure functions. Of course, the dlog forms for MHV amplitudes cannot be integrated directly in four dimensions, but dimensional regularisation is not a priori incompatible with a similar analysis of transcendental behaviour. For example, it was mentioned in [84] that dlog forms can naturally accommodate additional factors needed in dimensional regularisation, leading to results of uniform transcendental weight. We have demonstrated by our explicit reduction that they are indeed of uniform transcendental weight in $D=4-2 \epsilon$ dimensions for all $n$, but already the case $n=6$ shows that they do not satisfy the stronger condition of purity.

\section{Conclusion and outlook}

In this article we have proved the dimension-shift conjecture in equation (1.1) by using unitarity cuts with a massive spinor-helicity formalism. We have also demonstrated how to compute the one-loop amplitudes in the conjecture to all orders in epsilon and at all multiplicities. This is facilitated by the simplicity of the pentagon cut solution (3.26).

Although one-loop amplitudes have for the most part long been computable to any extent required by phenomenological applications, these results demonstrate that these two simple classes of amplitude can be understood at a level of completeness not previously realised. Extending these results to other helicity configurations is also achievable, by including dimension-shifted bubble and triangle cuts and using a limiting procedure to resolve the extra unfixed parameters in the cut constraint [42, 59]. For any given configuration, one would also need the appropriate lower-point tree amplitudes with two massive legs.

Applying similar techniques to multi-loop amplitudes requires a general understanding of how to fix a basis as was done in section 3.1. We hope the one-loop techniques consolidated and developed in this work can provide insight and guidance into pushing understanding of multi-loop amplitudes beyond their current level.

Moreover, the theorem (1.1) and the proof given here bridges $\mathcal{N}=4$ super-Yang-Mills, with its remarkable simplicity, and more realistic theories like QCD. We hope that further simplifications and unifications of this kind follow down the line.

\section{Acknowledgments}

We would like to thank Simon Badger, David Dunbar, Riccardo Gonzo, Martijn Hidding, Robert Schabinger, and Johann Usovitsch for many helpful discussions. Diagrams were drawn using Axodraw. This work was supported by the European Research Council through grant number 647356 (CutLoops).

Open Access. This article is distributed under the terms of the Creative Commons Attribution License (CC-BY 4.0), which permits any use, distribution and reproduction in any medium, provided the original author(s) and source are credited. 


\section{References}

[1] S. Caron-Huot, L.J. Dixon, F. Dulat, M. Von Hippel, A.J. McLeod and G. Papathanasiou, The Cosmic Galois Group and Extended Steinmann Relations for Planar $\mathcal{N}=4 S Y M$ Amplitudes, JHEP 09 (2019) 061 [arXiv:1906.07116] [INSPIRE].

[2] L.J. Dixon and Y.-T. Liu, Lifting Heptagon Symbols to Functions, JHEP 10 (2020) 031 [arXiv:2007.12966] [INSPIRE].

[3] H. Elvang and Y.-t. Huang, Scattering Amplitudes in Gauge Theory and Gravity, Cambridge University Press, Cambridge U.K. (2015) [INSPIRE].

[4] N. Arkani-Hamed, J.L. Bourjaily, F. Cachazo, A.B. Goncharov, A. Postnikov and J. Trnka, Grassmannian Geometry of Scattering Amplitudes, Cambridge University Press, Cambridge U.K. (2016) [https://doi.org/10.1017/CBO9781316091548] [arXiv: 1212.5605] [INSPIRE].

[5] Z. Bern, L.J. Dixon, D.C. Dunbar and D.A. Kosower, One loop gauge theory amplitudes with an arbitrary number of external legs, in proceedings of the Workshop on Continuous Advances in QCD, Minneapolis, MN, U.S.A., 18-20 February 1994, pp. 3-21 [hep-ph/9405248] [inSPIRE].

[6] Z. Bern, L.J. Dixon, D.C. Dunbar and D.A. Kosower, Fusing gauge theory tree amplitudes into loop amplitudes, Nucl. Phys. B 435 (1995) 59 [hep-ph/9409265] [INSPIRE].

[7] R. Britto, F. Cachazo and B. Feng, Generalized unitarity and one-loop amplitudes in $N=4$ super-Yang-Mills, Nucl. Phys. B 725 (2005) 275 [hep-th/0412103] [INSPIRE].

[8] S. Caron-Huot and K.J. Larsen, Uniqueness of two-loop master contours, JHEP 10 (2012) 026 [arXiv: 1205.0801] [INSPIRE].

[9] S. Badger, H. Frellesvig and Y. Zhang, A Two-Loop Five-Gluon Helicity Amplitude in QCD, JHEP 12 (2013) 045 [arXiv:1310.1051] [INSPIRE].

[10] S. Badger, C. Brønnum-Hansen, H.B. Hartanto and T. Peraro, First look at two-loop five-gluon scattering in QCD, Phys. Rev. Lett. 120 (2018) 092001 [arXiv:1712.02229] [INSPIRE].

[11] S. Abreu, F. Febres Cordero, H. Ita, B. Page and M. Zeng, Planar Two-Loop Five-Gluon Amplitudes from Numerical Unitarity, Phys. Rev. D 97 (2018) 116014 [arXiv:1712.03946] [INSPIRE].

[12] T. Peraro, Scattering amplitudes over finite fields and multivariate functional reconstruction, JHEP 12 (2016) 030 [arXiv: 1608.01902] [INSPIRE].

[13] S. Badger, C. Brønnum-Hansen, H.B. Hartanto and T. Peraro, Analytic helicity amplitudes for two-loop five-gluon scattering: the single-minus case, JHEP 01 (2019) 186 [arXiv: 1811.11699] [INSPIRE].

[14] S. Abreu, J. Dormans, F. Febres Cordero, H. Ita and B. Page, Analytic Form of Planar Two-Loop Five-Gluon Scattering Amplitudes in QCD, Phys. Rev. Lett. 122 (2019) 082002 [arXiv: 1812.04586] [INSPIRE].

[15] S. Abreu, J. Dormans, F. Febres Cordero, H. Ita, B. Page and V. Sotnikov, Analytic Form of the Planar Two-Loop Five-Parton Scattering Amplitudes in QCD, JHEP 05 (2019) 084 [arXiv: 1904.00945] [INSPIRE].

[16] T. Gehrmann, J.M. Henn and N.A. Lo Presti, Analytic form of the two-loop planar five-gluon all-plus-helicity amplitude in QCD, Phys. Rev. Lett. 116 (2016) 062001 [Erratum ibid. 116 (2016) 189903] [arXiv: 1511.05409] [INSPIRE]. 
[17] D.C. Dunbar and W.B. Perkins, Two-loop five-point all plus helicity Yang-Mills amplitude, Phys. Rev. D 93 (2016) 085029 [arXiv:1603.07514] [InSPIRE].

[18] D.C. Dunbar, J.H. Godwin, G.R. Jehu and W.B. Perkins, Analytic all-plus-helicity gluon amplitudes in QCD, Phys. Rev. D 96 (2017) 116013 [arXiv:1710.10071] [INSPIRE].

[19] S. Badger et al., Analytic form of the full two-loop five-gluon all-plus helicity amplitude, Phys. Rev. Lett. 123 (2019) 071601 [arXiv:1905.03733] [INSPIRE].

[20] D.C. Dunbar, J.H. Godwin, W.B. Perkins and J.M.W. Strong, Color Dressed Unitarity and Recursion for Yang-Mills Two-Loop All-Plus Amplitudes, Phys. Rev. D 101 (2020) 016009 [arXiv: 1911.06547] [INSPIRE].

[21] J. Henn, B. Power and S. Zoia, Conformal Invariance of the One-Loop All-Plus Helicity Scattering Amplitudes, JHEP 02 (2020) 019 [arXiv:1911.12142] [INSPIRE].

[22] D.C. Dunbar, W.B. Perkins and J.M.W. Strong, n-point QCD two-loop amplitude, Phys. Rev. D 101 (2020) 076001 [arXiv:2001.11347] [INSPIRE].

[23] Z. Bern, L.J. Dixon, D.C. Dunbar and D.A. Kosower, One loop selfdual and $N=4$ superYang-Mills, Phys. Lett. B 394 (1997) 105 [hep-th/9611127] [InSPIRE].

[24] D. Cangemi, Selfduality and maximally helicity violating QCD amplitudes, Int. J. Mod. Phys. A 12 (1997) 1215 [hep-th/9610021] [INSPIRE].

[25] G. Chalmers and W. Siegel, The Selfdual sector of QCD amplitudes, Phys. Rev. D 54 (1996) 7628 [hep-th/9606061] [INSPIRE].

[26] W.A. Bardeen, Selfdual Yang-Mills theory, integrability and multiparton amplitudes, Prog. Theor. Phys. Suppl. 123 (1996) 1 [inSPIRE].

[27] P. Chattopadhyay and K. Krasnov, One-loop same helicity four-point amplitude from shifts, JHEP 06 (2020) 082 [arXiv:2002.11390] [INSPIRE].

[28] A.D. Popov, Selfdual Yang-Mills: Symmetries and moduli space, Rev. Math. Phys. 11 (1999) 1091 [hep-th/9803183] [INSPIRE].

[29] H. Ooguri and C. Vafa, All loop $N=2$ string amplitudes, Nucl. Phys. B 451 (1995) 121 [hep-th/9505183] [INSPIRE].

[30] M.B. Green, J.H. Schwarz and L. Brink, $N=4$ Yang-Mills and $N=8$ Supergravity as Limits of String Theories, Nucl. Phys. B 198 (1982) 474 [INSPIRE].

[31] Z. Bern and D.A. Kosower, The Computation of loop amplitudes in gauge theories, Nucl. Phys. B 379 (1992) 451 [inSPIRE].

[32] Z. Bern and D.C. Dunbar, A Mapping between Feynman and string motivated one loop rules in gauge theories, Nucl. Phys. B 379 (1992) 562 [INSPIRE].

[33] N. Ahmadiniaz and C. Schubert, Off-shell Ward identities for N-gluon amplitudes, Europhys. Lett. 130 (2020) 41001 [arXiv: 2001.00885] [INSPIRE].

[34] N. Ahmadiniaz, V.M. Banda Guzmán, F. Bastianelli, O. Corradini, J.P. Edwards and C. Schubert, Worldline master formulas for the dressed electron propagator. Part I. Off-shell amplitudes, JHEP 08 (2020) 018 [arXiv:2004.01391] [INSPIRE].

[35] D. Bonocore, Asymptotic dynamics on the worldline for spinning particles, JHEP 02 (2021) 007 [arXiv: 2009. 07863] [inSPIRE].

[36] G. Mogull, J. Plefka and J. Steinhoff, Classical black hole scattering from a worldline quantum field theory, JHEP 02 (2021) 048 [arXiv:2010.02865] [INSPIRE]. 
[37] R.M. Schabinger, One-Loop $N=4$ Super Yang-Mills Scattering Amplitudes to All Orders in the Dimensional Regularization Parameter, arXiv:1103.2769 [INSPIRE].

[38] S. Stieberger and T.R. Taylor, Amplitude for N-Gluon Superstring Scattering, Phys. Rev. Lett. 97 (2006) 211601 [hep-th/0607184] [INSPIRE].

[39] M.T. Grisaru and H.N. Pendleton, Some Properties of Scattering Amplitudes in Supersymmetric Theories, Nucl. Phys. B 124 (1977) 81 [INSPIRE].

[40] L.J. Dixon, Calculating scattering amplitudes efficiently, in proceedings of the Theoretical Advanced Study Institute in Elementary Particle Physics (TASI 95): QCD and Beyond, Boulder, CO, U.S.A., 4-30 June 1995, hep-ph/9601359 [INSPIRE].

[41] Z. Bern, String based perturbative methods for gauge theories, in proceedings of the Theoretical Advanced Study Institute (TASI 92): From Black Holes and Strings to Particles, Boulder, CO, U.S.A., 3-28 June 1992, hep-ph/9304249 [INSPIRE].

[42] S.D. Badger, Direct Extraction Of One Loop Rational Terms, JHEP 01 (2009) 049 [arXiv:0806.4600] [INSPIRE].

[43] Z. Bern, L.J. Dixon and D.A. Kosower, Dimensionally regulated pentagon integrals, Nucl. Phys. B 412 (1994) 751 [hep-ph/9306240] [INSPIRE].

[44] G.R. Jehu, Symmetric reduction of high-multiplicity one-loop integrals and maximal cuts, JHEP 02 (2021) 013 [arXiv:2010.16266] [INSPIRE].

[45] S. Abreu, R. Britto, C. Duhr and E. Gardi, Cuts from residues: the one-loop case, JHEP 06 (2017) 114 [arXiv: 1702.03163] [InSPIRE].

[46] M. Bianchi, H. Elvang and D.Z. Freedman, Generating Tree Amplitudes in $N=4$ SYM and $N=8$ SG, JHEP 09 (2008) 063 [arXiv:0805.0757] [inSPIRE].

[47] G. Mahlon, Multi-gluon helicity amplitudes involving a quark loop, Phys. Rev. D 49 (1994) 4438 [hep-ph/9312276] [INSPIRE].

[48] Z. Bern, G. Chalmers, L.J. Dixon and D.A. Kosower, One loop $N$ gluon amplitudes with maximal helicity violation via collinear limits, Phys. Rev. Lett. 72 (1994) 2134 [hep-ph/9312333] [INSPIRE].

[49] Z. Bern and A.G. Morgan, Massive loop amplitudes from unitarity, Nucl. Phys. B 467 (1996) 479 [hep-ph/9511336] [INSPIRE].

[50] S. Dittmaier, Weyl-van der Waerden formalism for helicity amplitudes of massive particles, Phys. Rev. D 59 (1998) 016007 [hep-ph/9805445] [INSPIRE].

[51] N. Arkani-Hamed, T.-C. Huang and Y.-t. Huang, Scattering Amplitudes For All Masses and Spins, arXiv:1709.04891 [INSPIRE].

[52] R.H. Boels and C. Schwinn, On-shell supersymmetry for massive multiplets, Phys. Rev. D 84 (2011) 065006 [arXiv: 1104.2280] [INSPIRE].

[53] N. Craig, H. Elvang, M. Kiermaier and T.R. Slatyer, Massive amplitudes on the Coulomb branch of $N=4$ SYM, JHEP 12 (2011) 097 [arXiv:1104.2050] [INSPIRE].

[54] M. Kiermaier, The Coulomb-branch S-matrix from massless amplitudes, arXiv:1105.5385 [INSPIRE].

[55] H. Elvang, D.Z. Freedman and M. Kiermaier, Integrands for QCD rational terms and $N=4$ SYM from massive CSW rules, JHEP 06 (2012) 015 [arXiv:1111.0635] [INSPIRE].

[56] R.J. Eden, P.V. Landshoff, D.I. Olive and J.C. Polkinghorne, The analytic S-matrix, Cambridge University Press, Cambridge U.K. (1966) [InSPIRE]. 
[57] Z. Bern, L.J. Dixon, D.C. Dunbar and D.A. Kosower, One loop $n$ point gauge theory amplitudes, unitarity and collinear limits, Nucl. Phys. B 425 (1994) 217 [hep-ph/9403226] [INSPIRE].

[58] R. Britto, B. Feng and P. Mastrolia, The Cut-constructible part of QCD amplitudes, Phys. Rev. D 73 (2006) 105004 [hep-ph/0602178] [INSPIRE].

[59] D. Forde, Direct extraction of one-loop integral coefficients, Phys. Rev. D 75 (2007) 125019 [arXiv:0704.1835] [INSPIRE].

[60] D.C. Dunbar, W.B. Perkins and E. Warrick, The Unitarity Method using a Canonical Basis Approach, JHEP 06 (2009) 056 [arXiv:0903.1751] [INSPIRE].

[61] P. Mastrolia, Double-Cut of Scattering Amplitudes and Stokes' Theorem, Phys. Lett. B 678 (2009) 246 [arXiv: 0905. 2909] [INSPIRE].

[62] Z. Bern, L.J. Dixon and D.A. Kosower, On-shell recurrence relations for one-loop QCD amplitudes, Phys. Rev. D 71 (2005) 105013 [hep-th/0501240] [InSPIRE].

[63] D.C. Dunbar, J.H. Ettle and W.B. Perkins, Augmented Recursion For One-loop Amplitudes, Nucl. Phys. B Proc. Suppl. 205-206 (2010) 74 [arXiv:1011.0559] [inSPIRE].

[64] S.D. Alston, D.C. Dunbar and W.B. Perkins, Complex Factorisation and Recursion for One-Loop Amplitudes, Phys. Rev. D 86 (2012) 085022 [arXiv:1208.0190] [INSPIRE].

[65] W.T. Giele, Z. Kunszt and K. Melnikov, Full one-loop amplitudes from tree amplitudes, JHEP 04 (2008) 049 [arXiv:0801.2237] [INSPIRE].

[66] R.K. Ellis, W.T. Giele, Z. Kunszt and K. Melnikov, Masses, fermions and generalized D-dimensional unitarity, Nucl. Phys. B $\mathbf{8 2 2}$ (2009) 270 [arXiv:0806.3467] [InSPIRE].

[67] G. Ossola, C.G. Papadopoulos and R. Pittau, On the Rational Terms of the one-loop amplitudes, JHEP 05 (2008) 004 [arXiv:0802.1876] [INSPIRE].

[68] C. Cheung and D. O'Connell, Amplitudes and Spinor-Helicity in Six Dimensions, JHEP 07 (2009) 075 [arXiv:0902.0981] [INSPIRE].

[69] Z. Bern, J.J. Carrasco, T. Dennen, Y.-t. Huang and H. Ita, Generalized Unitarity and Six-Dimensional Helicity, Phys. Rev. D 83 (2011) 085022 [arXiv: 1010.0494] [InSPIRE].

[70] S. Badger, C. Brønnum-Hansen, F. Buciuni and D. O'Connell, A unitarity compatible approach to one-loop amplitudes with massive fermions, JHEP 06 (2017) 141 [arXiv: 1703.05734] [INSPIRE].

[71] W. Pauli and F. Villars, On the Invariant regularization in relativistic quantum theory, Rev. Mod. Phys. 21 (1949) 434 [inSPIRE].

[72] D. Forde and D.A. Kosower, All-multiplicity amplitudes with massive scalars, Phys. Rev. D 73 (2006) 065007 [hep-th/0507292] [INSPIRE].

[73] G. Rodrigo, Multigluonic scattering amplitudes of heavy quarks, JHEP 09 (2005) 079 [hep-ph/0508138] [INSPIRE].

[74] P. Ferrario, G. Rodrigo and P. Talavera, Compact multigluonic scattering amplitudes with heavy scalars and fermions, Phys. Rev. Lett. 96 (2006) 182001 [hep-th/0602043] [INSPIRE].

[75] A. Ochirov, Helicity amplitudes for QCD with massive quarks, JHEP 04 (2018) 089 [arXiv: 1802.06730] [INSPIRE].

[76] C. Schwinn and S. Weinzierl, SUSY ward identities for multi-gluon helicity amplitudes with massive quarks, JHEP 03 (2006) 030 [hep-th/0602012] [INSPIRE]. 
[77] C. Schwinn and S. Weinzierl, Scalar diagrammatic rules for Born amplitudes in QCD, JHEP 05 (2005) 006 [hep-th/0503015] [INSPIRE].

[78] G. Passarino and M.J.G. Veltman, One Loop Corrections for $e^{+} e^{-}$Annihilation Into $\mu^{+} \mu^{-}$ in the Weinberg Model, Nucl. Phys. B 160 (1979) 151 [INSPIRE].

[79] W.L. van Neerven and J.A.M. Vermaseren, Large loop integrals, Phys. Lett. B 137 (1984) 241 [INSPIRE].

[80] D.B. Melrose, Reduction of Feynman diagrams, Nuovo Cim. 40 (1965) 181 [InSPIRE].

[81] N. Arkani-Hamed, J.L. Bourjaily, F. Cachazo and J. Trnka, Local Integrals for Planar Scattering Amplitudes, JHEP 06 (2012) 125 [arXiv:1012.6032] [INSPIRE].

[82] S. Abreu, R. Britto, C. Duhr and E. Gardi, From multiple unitarity cuts to the coproduct of Feynman integrals, JHEP 10 (2014) 125 [arXiv:1401.3546] [INSPIRE].

[83] S. Caron-Huot, Superconformal symmetry and two-loop amplitudes in planar $N=4$ super Yang-Mills, JHEP 12 (2011) 066 [arXiv:1105.5606] [INSPIRE].

[84] J.M. Henn, Lectures on differential equations for Feynman integrals, J. Phys. A 48 (2015) 153001 [arXiv: 1412.2296] [INSPIRE]. 